\title{
c-Jun N-terminal kinase 2 promotes enterocyte survival and goblet cell differentiation in the inflamed intestine
}

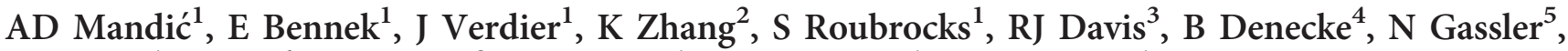 \\ K Streetz ${ }^{1}$, A Kel ${ }^{6}$, M Hornef $^{2}$, FJ Cubero ${ }^{1}$, C Trautwein ${ }^{1}$ and G Sellge ${ }^{1}$
}

C-Jun N-terminal kinases (JNKs) contribute to immune signaling but their functional role during intestinal mucosal inflammation has remained ill defined. Using genetic mouse models, we characterized the role of JNK1 and JNK2 during homeostasis and acute colitis. Epithelial apoptosis, regeneration, differentiation, and barrier function were analyzed in intestinal epithelium-specific ( $\triangle \mathrm{IEC}$ ) or complete JNK1 and bone marrow chimeric or complete JNK2 deficient mice as well as double-knockout animals (JNK1 $1^{\Delta \mathrm{IEC}} \mathrm{JNK} 2^{-{ }^{-}}$) during homeostasis and acute dextran sulfate sodium (DSS)induced colitis. Results were confirmed using human HT-29 cells and wild-type or JNK2-deficient mouse intestinal organoid cultures. We show that nonhematopoietic JNK2 but not JNK1 expression confers protection from DSSinduced intestinal inflammation reducing epithelial barrier dysfunction and enterocyte apoptosis. JNK2 additionally enhanced Atonal homolog 1 expression, goblet cell and enteroendocrine cell differentiation, and mucus production under inflammatory conditions. Our results identify a protective role of epithelial JNK2 signaling to maintain mucosal barrier function, epithelial cell integrity, and mucus layer production in the event of inflammatory tissue damage.

\section{INTRODUCTION}

c-Jun N-terminal kinases (JNK) are members of the mitogen-activated protein-kinase family. They are activated in intestinal epithelial cells (IECs) and immune cells under inflammatory conditions such as in patients suffering from inflammatory bowel disease (IBD). ${ }^{1,2,3}$ Previous reports suggest an important role of JNKs to maintain intestinal homeostasis during gut inflammation. ${ }^{4}$ Several IBD risk loci represent genes that act upstream of JNKs such as NOD2 (nucleotide-binding oligomerization domain containing 2), TNFSF9/15 (tumor necrosis factor (ligand) superfamily, member 9 and 15), and MAP3K8 (mitogen-activated protein kinase kinase kinase 8$){ }^{5-9}$

JNKs are expressed from three genes, Jnk1, Jnk2, and Jnk3, each generating 2-4 splice variants. This results in up to 10 different isoforms varying in size between 46 and $55 \mathrm{kDa}$, which have overlapping and also distinct biological functions. JNK1 and JNK2 are ubiquitously expressed, whereas the expression of JNK3 is restricted to the brain, heart, and testis. JNKs are activated in response to multiple stimuli such as growth factors, endogenous, and exogenous inflammatory stimuli as well as extracellular and intracellular stress. ${ }^{10,11}$ Subsequently, JNKs phosphorylate multiple downstream targets. In addition to the transcription factor c-Jun, more than 50 nuclear and cytoplasmic JNK substrates have been identified; among them are several transcription factors, nuclear hormone receptors, mitochondrial proteins, and protein kinases. ${ }^{12}$ JNKs have an important role in a variety of biological processes including cell proliferation, differentiation, apoptosis, regulation of intercellular adhesion, and immune cell regulation.

The downstream effects of JNK activation depend on the stimulus, cell type, and physiological condition and hence the ultimate influence on organ function and disease severity during enteric tissue inflammation cannot be predicted. For

${ }^{1}$ Department of Internal Medicine III, University Hospital RWTH Aachen, Aachen, Germany. ${ }^{2}$ Department of Microbiology, University Hospital RWTH Aachen, Aachen, Germany. ${ }^{3}$ Howard Hughes Medical Institute and University of Massachusetts Medical School, Worcester, Massachusetts, USA. ${ }^{4}$ Interdisciplinary Center for Clinical Research (IZKF), University Hospital RWTH Aachen, Aachen, Germany. ${ }^{5}$ Institute of Pathology, Klinikum Braunschweig, Braunschweig, Germany and ${ }^{6}$ GeneXplain GmbH, Wolfenbüttel, Germany. Correspondence: G Sellge (gsellge@ukaachen.de) 
example, previous reports suggest that JNKs mediate the proapoptotic activity in multiple cell types and tissues. They directly target proapoptotic $\mathrm{Bcl}-2$ proteins and stimulate the transcription of proapoptotic genes such as Tnf, FasL, and $B a k \cdot{ }^{13}$ However, JNK have also been reported to exert antiapoptotic activity. Constitutional JNK1/JNK2 doubleknockout mice are lethal and embryos show strongly elevated apoptosis in the forebrain. ${ }^{14}$ Also, JNK1 promotes the survival of BCL/Abl-transformed B lymphoblasts, hepatocellular carcinoma cells, and fibroblasts by increasing anti-apoptotic $\mathrm{Bcl} 2$ expression, ${ }^{15}$ down regulating $\mathrm{p} 21,{ }^{16}$ and inducing cIAP2 via JunD, ${ }^{17}$ respectively.

Several functions for JNKs have been proposed suggesting that JNK could either control or promote intestinal inflammation. JNK influences via c-Jun the Wnt pathway leading to intestinal progenitor cell proliferation and tissue regeneration. ${ }^{18,19}$ However, JNKs also induce proinflammatory genes in immune cells ${ }^{4}$ and suppress the expression of tight junction proteins that have a central role in maintaining the intestinal epithelial barrier. ${ }^{20,21}$ Administration of unselective JNK inhibitors $^{3,20,22}$ or JNK1-specific inhibitors ${ }^{22,23}$ reduced inflammation and tissue destruction in experimental colitis models. In contrast, increased weight loss, tissue destruction, and inflammatory cell infiltration was found in JNK1 - or JNK2deficient mice in dextran sulfate sodium (DSS)-induced chronic colitis. ${ }^{24,25}$

We hypothesized that cell-type-specific downstream effects and compensatory functions of JNK1 and JNK2 may explain previous conflicting results. We therefore analyzed mice with deletion of JNK1 or JNK2 in the epithelial or somatic cell compartment, respectively, in combination with a wellestablished chemical colitis model to elucidate the functional role of JNK signaling during homeostasis and intestinal inflammation.

\section{RESULTS}

\section{Colonic JNK expression in humans and mice}

Recent studies demonstrated elevated levels of phosphorylated JNK (p-JNK) in IECs and lamina propria (LP) cells in IBD patients, ${ }^{2,3}$ whereas Hommes et al. showed increased p-JNK expression only in LP cells of Crohn's disease (CD) patients. ${ }^{1}$ We found that p-JNK was predominantly localized in the cytoplasm and nuclei of IECs at the luminal side, but was also detectable in LP cells in patients with active ulcerative colitis (UC) and CD, but not in noninflamed tissue from control patients (Figure 1a).

Next, we studied mice with a conditional IEC-specific JNK1 $\left(\mathrm{JNK} 1^{\Delta \mathrm{IEC}}\right.$ ), a complete JNK2 (JNK2 ${ }^{-/-}$), and a combined deletion (JNK1 ${ }^{\triangle \mathrm{IEC}} \mathrm{JNK} 2^{-1-}$ ) and compared their phenotypes with wild-type (WT) mice. Similar to the staining pattern in humans, p-JNK was undetectable by immunohistochemistry (IHC) in noninflamed colon, but was readily detectable upon colitis induction in IECs and LP cells of WT and JNK1 $1^{\Delta \mathrm{IEC}}$ mice, whereas p-JNK was almost undetectable in JNK2 $2^{-1-}$ and JNK1 ${ }^{\Delta \mathrm{IEC}} \mathrm{JNK} 2^{-1-}$ animals (Supplementary Figure 2B online). Western blot (WB) analyses of isolated IECs showed that total JNK was absent in JNK1 $1^{\Delta \mathrm{IEC}} \mathrm{JNK} 2^{-/-}$mice, strongly reduced in JNK2 ${ }^{-1-}$ mice, but only slightly diminished in $\mathrm{JNK1}^{\triangle \mathrm{IEC}}$ mice compared with WT mice, indicating a predominant expression of JNK2 in IECs (Figure 1b). p-JNK were detectable in WT mice by WB (in contrast to IHC) in the absence of intestinal inflammation but was further induced after colitis induction. p-JNK was partially reduced in $J N K 1^{\triangle I E C}$ and JNK2 ${ }^{-1-}$ mice, whereas the reduction was more pronounced in JNK2 ${ }^{-1-}$ mice. p-JNK was completely absent in $\mathrm{JNK}_{1}{ }^{\triangle \mathrm{IEC}} \mathrm{JNK} 2^{-1-}$ mice (Figure $\mathbf{1 b}, \mathbf{c}$ ).

We further analyzed the phosphorylated forms of the JNK downstream transcription factors c-Jun and JunD. p-c-Jun and p-JunD were strongly reduced, but not completely absent in IECs derived from JNK1 ${ }^{\Delta \mathrm{IEC}} \mathrm{JNK} 2^{-1-}$ mice. p-c-Jun and $\mathrm{p}$-JunD signals in JNK2 ${ }^{-1-}$ mice were comparable to WT mice, whereas in JNK1 ${ }^{\triangle \mathrm{IEC}}$ mice $\mathrm{p}$-c-Jun and $\mathrm{p}$-JunD levels were reduced, although less pronounced than in doubleknockout mice (Figure 1b,c). These results suggest a more important role of JNK1 in phosphorylating c-Jun and JunD. However, JNK-independent pathways may contribute to c-Jun and JunD activation in IECs, although we cannot completely exclude that the residual p-c-Jun and p-JunD signals in $\mathrm{JNK}^{\triangle \mathrm{IEC}} \mathrm{JNK}^{-1-}$ mice derived from contaminating cells.

\section{Small intestinal villus shortening in JNK2-deficient mice}

Constitutional JNK1/JNK2 double-knockout mice are embryonically lethal ${ }^{14}$ however, JNK1 ${ }^{\Delta \mathrm{IEC}}, \mathrm{JNK} 2^{-1-}$, and $\mathrm{JNK}^{\triangle \mathrm{IEC}}{ }_{\mathrm{JNK}} 2^{-1-}$ mice developed as WT mice with no significant differences in body weight, stool consistency, fertility, and in general appearance up to the age of 45 weeks (Supplementary Figure 3A and data not shown). Colon morphology was not affected by either genotype at 10 weeks (Figure 2a) or 45 weeks of age (Supplementary Figure 3A). Length of ileal villi were significantly decreased in JNK2 ${ }^{-1-}$ and JNK1 ${ }^{\triangle \mathrm{IEC}} \mathrm{JNK} 2^{-1-}$ mice compared with WT controls, whereas no further histological alterations in the ileum were observed at 10 weeks (Figure 2,c) and 45 weeks of age (Supplementary Figure 3C). We observed no differences in ileal Paneth cell numbers, basal level of gut permeability measured by FITC-dextran uptake, and tissue myeloperoxidase (MPO) activity, indicating neutrophil infiltration between 10week-old knockout and WT mice (Supplementary Figure 3D and data not shown).

\section{JNK2 in nonhaematopoietic cells is a protective factor in acute DSS colitis}

To investigate the role of JNKs in intestinal inflammation, we orally administered DSS for 7 days to induce acute colitis. The disease activity index (DAI) and weight loss were more pronounced in $\mathrm{JNK} 2^{-1-}$ and $\mathrm{JNK} 1^{\triangle \mathrm{IEC}} \mathrm{JNK} 2^{-1-}$ mice compared with WT and $\mathrm{JNK}^{\triangle \mathrm{IEC}}$ animals (Figure 3a,b). JNK1 ${ }^{\triangle \mathrm{IEC}}{ }_{\mathrm{JNK}} 2^{-1-}$ mice showed a slightly enhanced disease phenotype compared with JNK2 ${ }^{-1-}$; however, the differences did not reach statistical significance (Figure 3a,b). Disease severity correlated with histological alterations, impaired intestinal barrier function, immune cell infiltration in the 

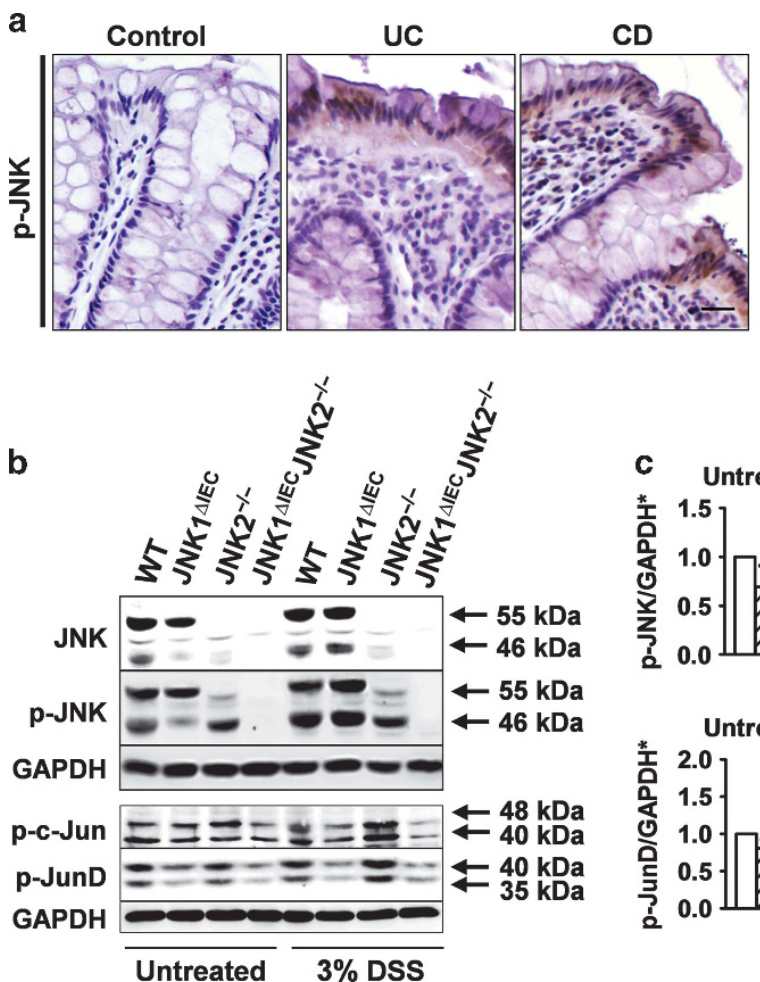
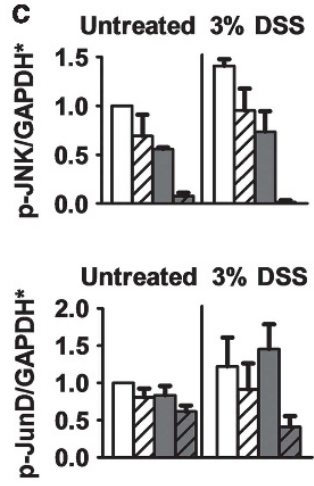

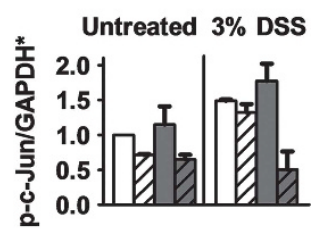

$\square$ WT

Z JNK1 ${ }^{\mathrm{AIEC}}$

$\square$ JNK2 $^{-1-}$

Z JNK1 ${ }^{\triangle \mathrm{IEC}}$ JNK2 $^{-I-}$

* Normalized to

WT untreated

Figure 1 Colonic c-Jun N-terminal kinase (JNK) expression in humans and mice. (a) Immunohistochemistry (IHC) detecting phosphorylated (p)-JNK1/ 2 in human colon tissue derived from patients with active ulcerative colitis (UC) and Crohn's disease (CD) and control patients without inflammation. $\mathrm{Bar}=20 \mu \mathrm{m}$. For negative control see Supplementary Figure 14. (b) Western blot (WB) analysis with antibodies (Abs) specific for p-JNK, total JNK, p-CJun, and p-JunD of colonic intestinal epithelial cell (IEC)-derived proteins isolated from untreated and 7 days dextran sulfate sodium (DSS)-treated wildtype (WT), JNK1 $1^{\Delta \mathrm{IEC}}, \mathrm{JNK} 2^{-l-}$ and JNK1 ${ }^{\Delta \mathrm{IEC}} \mathrm{JNK} 2^{-1-}$ mice. Forty-six and $54 \mathrm{kDa}$ bands indicate different JNK1/2 isotypes. Molecular weight of p-cJun and p-JunD isoforms are 40/48 and 35/40 kDa, respectively. Glyceraldehyde 3-phosphate dehydrogenase (GAPDH) was used as loading controls. (c) Densitometry analysis of three independent WB. Mean \pm s.e.m. of relative density normalized to GAPDH and the "WT untreated" condition is shown.
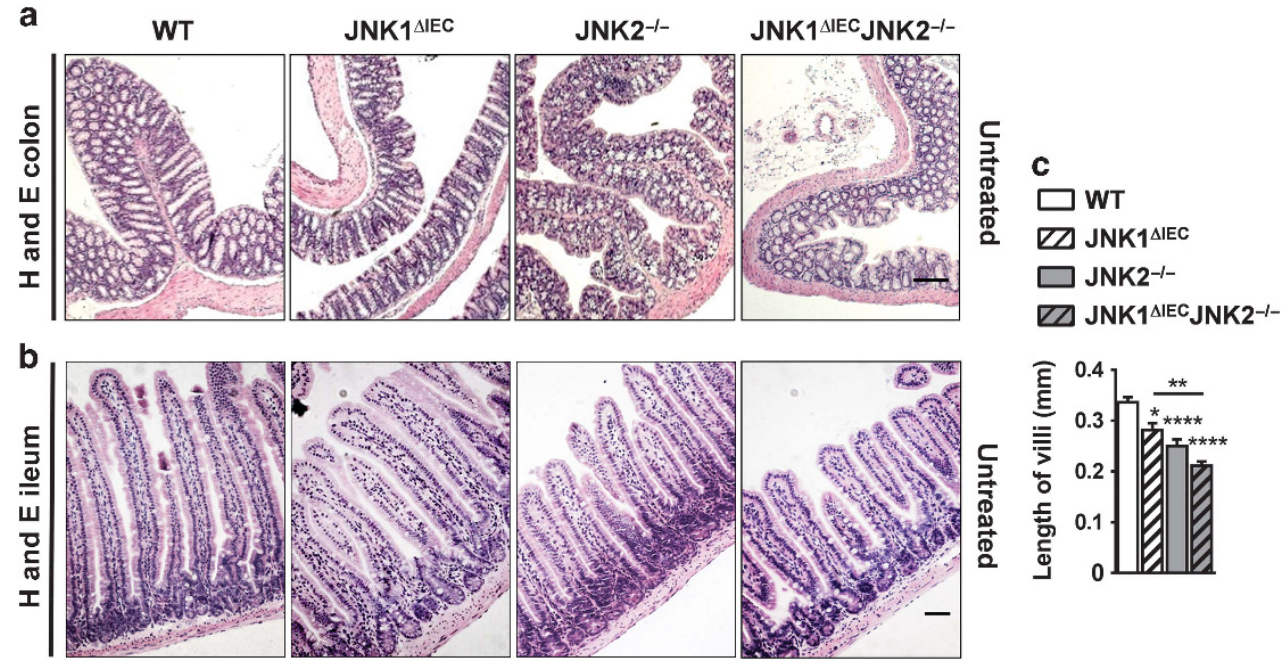

Figure 2 Small intestinal villus shortening in c-Jun N-terminal kinase 2 (JNK2)-deficient mice. (a and b) Representative hematoxylin and eosin (H\&E) images of (a) colon and (b) ileal sections of 10-week-old mice with indicated genotypes. Bar $=$ (a) $100 \mu \mathrm{m}$ and (b) $50 \mu \mathrm{m}$. (c) Graph shows length of the villi in the terminal ileum (means \pm s.e.m., $n=36$, nine villi in four mice per group, respectively). Statistics was performed with Kruskal-Wallis test and Dunn's post hoc analysis. ${ }^{\star} P<0.05 ;{ }^{*} P<0.01 ;{ }^{* * \star *} P<0.0001$. WT, wild type.

LP, and tissue mRNA expression of the proinflammatory cytokines Tnf and Illb (Figure 3c-g and Supplementary Figure 4).
To investigate whether JNK2 in hematopoietic or nonhematopoietic cells possess protective functions in DSS-induced colitis, we generated chimeric $\mathrm{WT} / \mathrm{JNK} 2^{-1-}$ mice by bone 

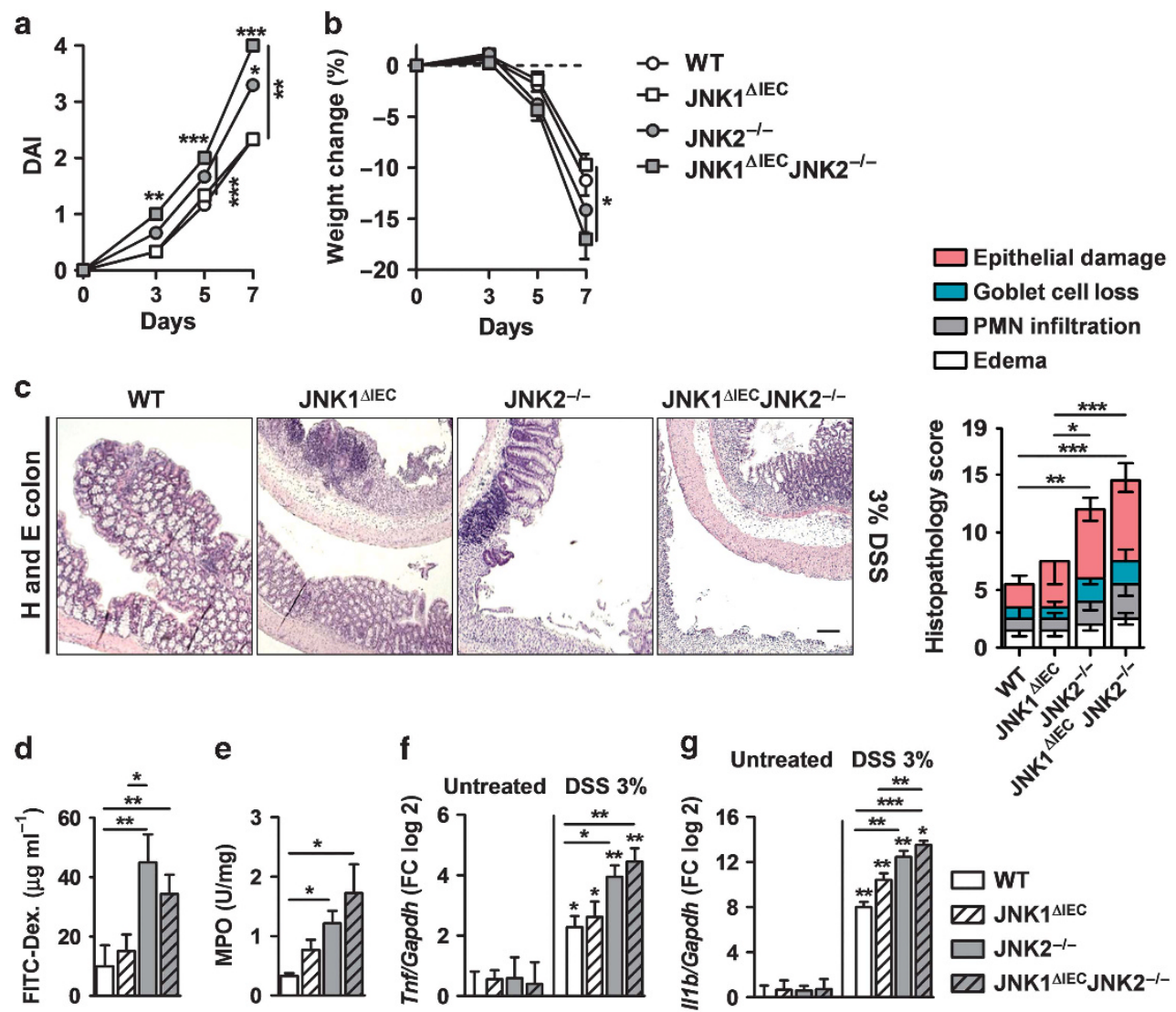

Figure 3 Jun N-terminal kinase 2 (JNK2)-deficient mice develop more severe dextran sulfate sodium (DSS) colitis. DSS colitis was induced in 8-10week-old wild-type (WT), JNK1 ${ }^{\Delta \mathrm{IEC}}$, JNK2 ${ }^{-l-}$, and JNK1 $1^{\Delta \mathrm{IEC}} \mathrm{JNK} 2^{-l-}$ mice $(n=12-18$ per group). (a) Medians (error bars are omitted for clarity) of disease activity scores (DAls) and (b) means \pm s.e.m. of weight changes at indicated time points are shown. (c) Representative hemotoxylin and eosin (H\&E) images of colon sections at day 7. Graph represents medians \pm interquartile ranges of histopathology scores. Bar $=100 \mu \mathrm{m}$. (d) Gut permeability measured by FITC-dextran tracer assays at day 6 (means \pm s.e.m.). (e) Myeloperoxidase (MPO) activity was measured in tissue lysates as an index of neutrophil infiltration at day 7 (means \pm s.e.m.). (f and $\mathbf{g}) T n f-\alpha$ and $/ 11 \beta$ mRNA expression in colonic tissue derived from untreated and 7 days DSS-treated WT, JNK1 $1^{\Delta \mathrm{IEC}}, \mathrm{JNK} 2^{-1-}$, and JNK1 $1^{\Delta \mathrm{IEC}} \mathrm{JNK} 2^{-1-}$ mice. Bars indicate mean \pm s.e.m. log 2 fold change (FC) compared with mean of WT untreated ( $n=4-8$ for untreated and $n=8-14$ for DSS-treated). Differences between genotypes were analyzed by Kruskal-Wallis and Dunn's post hoc test and are indicated by lines in (c-f). In (a and $\mathbf{b}$ ) asterisks above symbols indicate statistical differences compared with WT and further differences are indicated by lines. In ( $\mathbf{f}$ and $\mathbf{g}$ ) asterisks above bars indicate statistical differences between untreated and DSS-treated animals of the same genotype analyzed by Mann-Whitney $U$-test. ${ }^{\star} P<0.05 ;{ }^{\star \star} P<0.01 ;{ }^{\star \star \star} P<0.001$. IEC, intestinal epithelial cell; PMN, polymorphonuclear leukocyte.

marrow transplantation (BMT). Weight loss, DAI, MPO activity in colonic tissue, FITC-dextran uptake, histological scoring, and colonic Tnf and Il1b mRNA expression were significantly higher in $\mathrm{WT} \rightarrow \mathrm{JNK} 2^{-1-}$ and JNK2 ${ }^{-1-} \rightarrow \mathrm{JNK} 2^{-1-}$ animals compared with that in $\mathrm{WT} \rightarrow \mathrm{WT}$ and $\mathrm{JNK} 2^{-1-} \rightarrow \mathrm{WT}$ mice (Figure 4a-g and Supplementary Figure 5A). Histological analysis was confirmed by mouse endoscopy, showing more pronounced mucosal erythema, bleeding, erosions, and ulcerations in the colons of $\mathrm{WT} \rightarrow \mathrm{JNK} 2^{-1-}$ and $\mathrm{JNK}^{-1-}$ $\rightarrow \mathrm{JNK} 2^{-l-}$ mice (Figure 4h).

To exclude a role of JNK1 in non-IECs during acute colitis, we included constitutive JNK1 knockout mice in our analysis. No significant differences in DAI, weight loss, histopathology, and endoscopic score were observed between WT and JNK1 ${ }^{-1-}$ mice (Figure 4i-l and Supplementary Figure 5B and C).

Taken together, these results indicated that JNK2 in nonhematopoietic cells is a protective factor during acute DSS-induced colitis, whereas JNK1 in IECs and nonepithelial cells as well as JNK2 in hematopoietic cells have only minor relevance in this condition and cannot substitute the loss of JNK2 in nonhematopoietic cells.

\section{Regulation of tight junction proteins}

Several in vitro studies reported that JNK signaling downregulates the expression of tight junction and adherent junction proteins, which would suggest a negative influence of JNK signaling on intestinal barrier functions. However, in our in vivo experiments we detected increased barrier disruption during DSS colitis in the absence of JNK2 indicated by increased uptake of FITC-dextran (Figures $\mathbf{3 d}$ and $\mathbf{4 d}$ ) as well as decreased colonic expression of the tight junction protein zonula occludens protein-1 and occludin (Supplementary Figure 6). Barrier disruption might not be a direct effect of absent JNK2 signaling, but a consequence of enhanced inflammation and tissue disruption in JNK2deficient mice. 


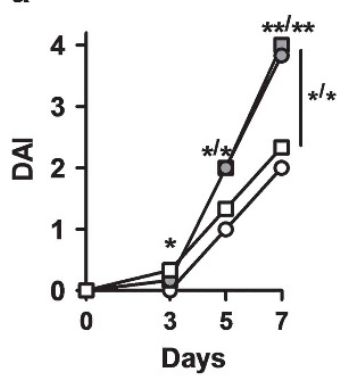

b

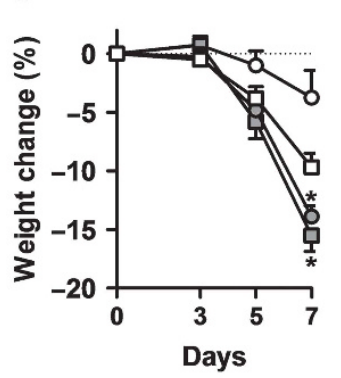

-o- WT $\rightarrow$ WT

$-\square-$ JNK2 $^{-1-} \rightarrow$ WT

- - WT $\rightarrow$ JNK2-I-

$\rightarrow \mathrm{JNK2}^{-1-} \rightarrow \mathrm{JNK2}^{-1-}$

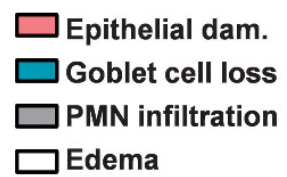

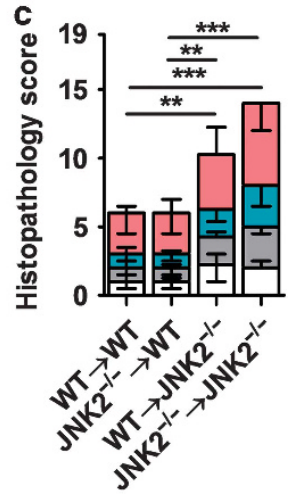

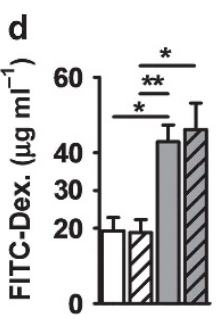

e

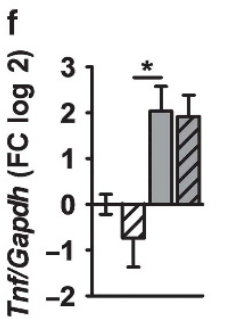

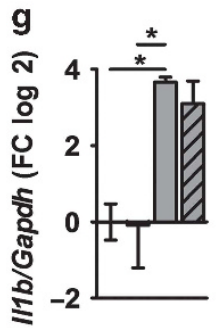

Legend for D - H

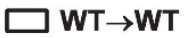

Z JNK2 ${ }^{-l-} \rightarrow$ WT

$\square$ WT $\rightarrow$ JNK2-I-

ZZ JNK2 $^{-I-} \rightarrow \mathrm{JNK2}^{-I-}$

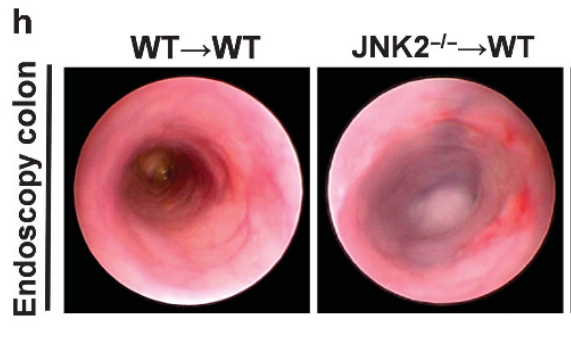

i

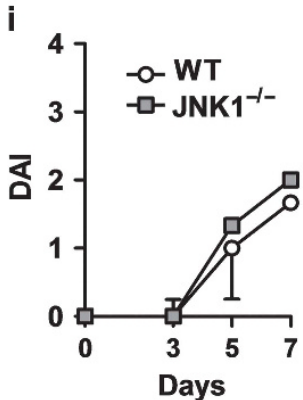

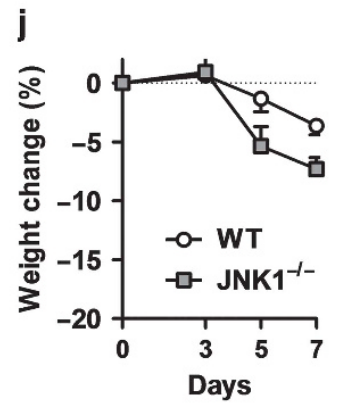
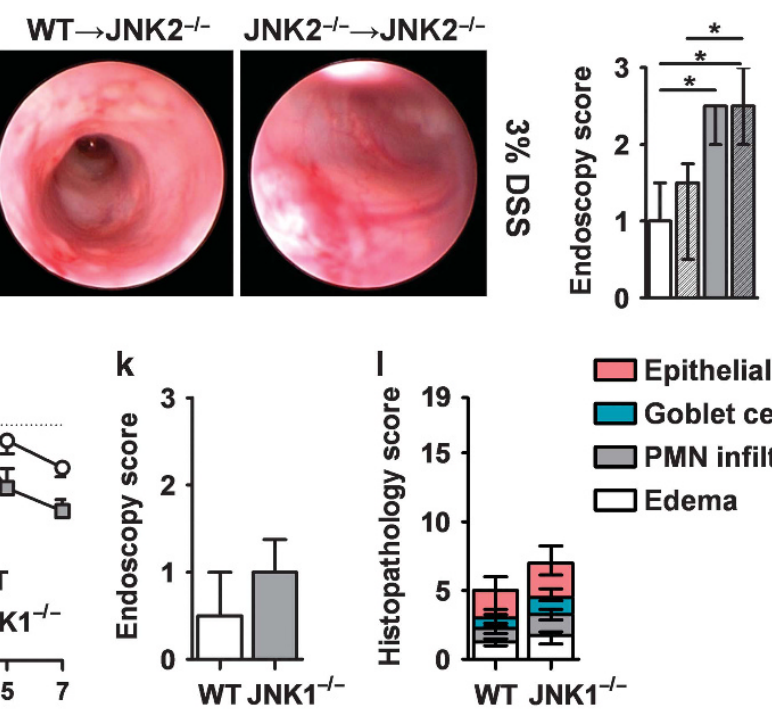

Figure 4 Jun N-terminal kinase 2 (JNK2) in nonhematopoietic cells protects against dextran sulfate sodium (DSS) colitis. (a-h) Bone marrow derived from wild-type (WT) and JNK2 ${ }^{-1-}$ mice were transplanted into WT and JNK2 ${ }^{-1-}$ to create chimeric and homogenous control mice $(n=5-8$ per group). Eight weeks after bone marrow transplantation (BMT) animals are subjected to 7 days of DSS treatment. (a) Medians (error bar are omitted for clarity) of disease activity score (DAI) and (b) mean \pm s.e.m. of weight changes at indicated time points. (c) Histopathology scores at day 7 (medians \pm interquartile ranges). (d) Changes in gut permeability measured by FITC-dextran tracer assays at day 6 (means \pm s.e.m.). (e) Myeloperoxidase (MPO) activity measured in tissue lysates at day 7 (means \pm s.e.m.). (f and $\mathbf{g}$ ) mRNA expression in colonic tissue for Tnf- $\alpha$ and $/ / 1 \beta$. Bars indicate mean \pm s.e.m. log 2 fold change (FC) compared with mean of WT $\rightarrow$ WT mice. (h) Endoscopic pictures of colons at day 7. Graph represents medians \pm interquartile ranges of endoscopic scores. (i-I) DSS colitis was induced in constitutive JNK1 ${ }^{-1}$ - and WT mice ( $n=4$ per group). (i) Medians \pm interquartile ranges of DAI and (j) mean \pm s.e.m. of weight changes at indicated time points. (k) Endoscopic scores at day 7 (median \pm interquartile range). (I) Histopathology score at day 7 (median \pm interquartile range). Statistics was performed with Kruskal-Wallis test and Dunn's post hoc analysis in (a-h) and Mann-Whitney $U$-test in (i-I). ${ }^{\star} P<0.05 ;{ }^{\star \star} P<0.01 ;{ }^{* \star *} P<0.001$. PMN, polymorphonuclear leukocyte.

\section{JNK2-deficient mice display increased IEC apoptosis and compensatory hyperproliferation}

Studies in mice with IEC-specific deletion of c-Jun and with IEC-specific overexpression of active JNK1 have shown that JNK-induced c-Jun signaling promotes intestinal stem cell differentiation and proliferation via cross-talk with the Wnt pathway. ${ }^{18,19}$ In JNK-deficient mice, we detected a tendency toward reduced colonic mRNA expression of the Wnt target genes Axin2, Lgr5, Tcf4, Cd44, and Jun (Supplementary Figure 7A), which however reached only statistical significance for Axin2 in $\mathrm{JNK}^{-1-}$ and $\mathrm{JNK} 1^{\triangle \mathrm{IEC}} \mathrm{JNK} 2^{-1-}$ mice. However, in the terminal ileum no difference in Wnt target gene expression was observed (Supplementary Figure 7B). In contrast to the studies using IEC-specific deletion of c-Jun, we 
found increased IEC proliferation in $\mathrm{JNK}^{-/-}$and $\mathrm{JNK}^{\Delta \mathrm{IEC}} \mathrm{JNK} 2^{-/-}$mice compared with WT animals. Elevated IEC proliferation in JNK2-deficient mice was detectable in untreated mice and further elevated during DSS colitis (Figure 5a,b and Supplementary Figure 7C). IEC proliferation was increased in mice with a JNK2 deletion in nonhematopoietic cells but not in mice with a JNK2 deletion in the hematopoietic compartment (Figure $\mathbf{5 c}$ and Supplementary Figure $7 \mathrm{D})$. In $\mathrm{JNK} 1^{\Delta \mathrm{IEC}}$ and $\mathrm{JNK} 1^{-/-}$ mice, we did not observe a significant difference in IEC proliferation compared with WT animals (Figure 5a,b,d and Supplementary Figure 7C and E).

We analyzed apoptosis in IECs by performing staining for cleaved caspase-3 and TUNNEL (terminal deoxynucleotidyl transferase dUTP nick-end labeling) assay and observed that IEC apoptosis correlated with proliferation rates. Enhanced apoptosis in IECs was detectable in $\mathrm{JNK}^{-/-}$and JNK1 ${ }^{\Delta \mathrm{IEC}} \mathrm{JNK} 2^{-/-}$mice already during homeostasis and was further enhanced during DSS colitis (Figure 5e,f,i and Supplementary Figure 8A,B). Increase in IEC apoptosis was
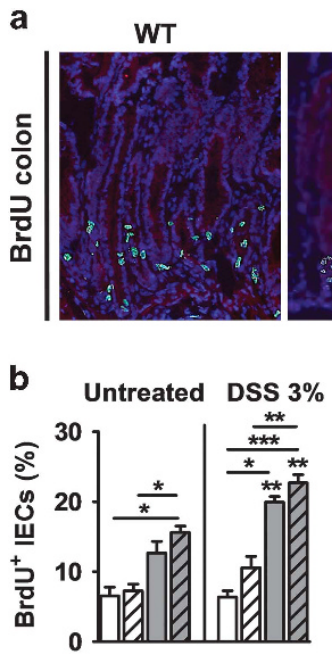

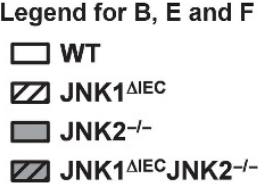

\section{JNK1 ${ }^{\triangle I E C}$}
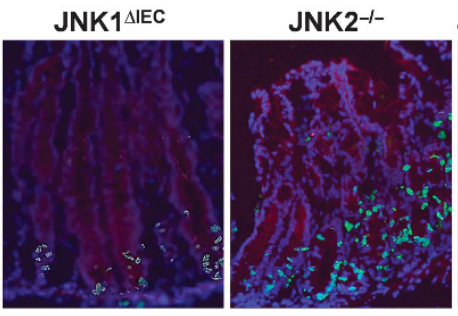

c

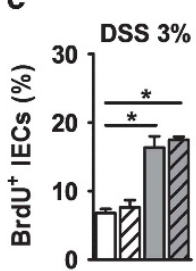

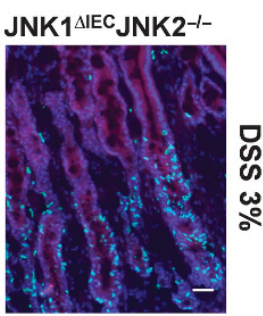

d

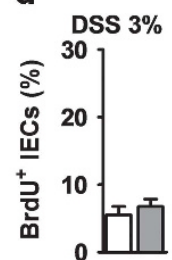

Legend for $\mathbf{C}$ and $\mathrm{G}$

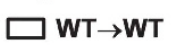

Z JNK2 ${ }^{-l-} \rightarrow$ WT

$\square \mathrm{WT} \rightarrow \mathrm{JNK}^{-1-}$

ZZ JNK2 ${ }^{-1-} \rightarrow$ JNK2 $^{-l-}$
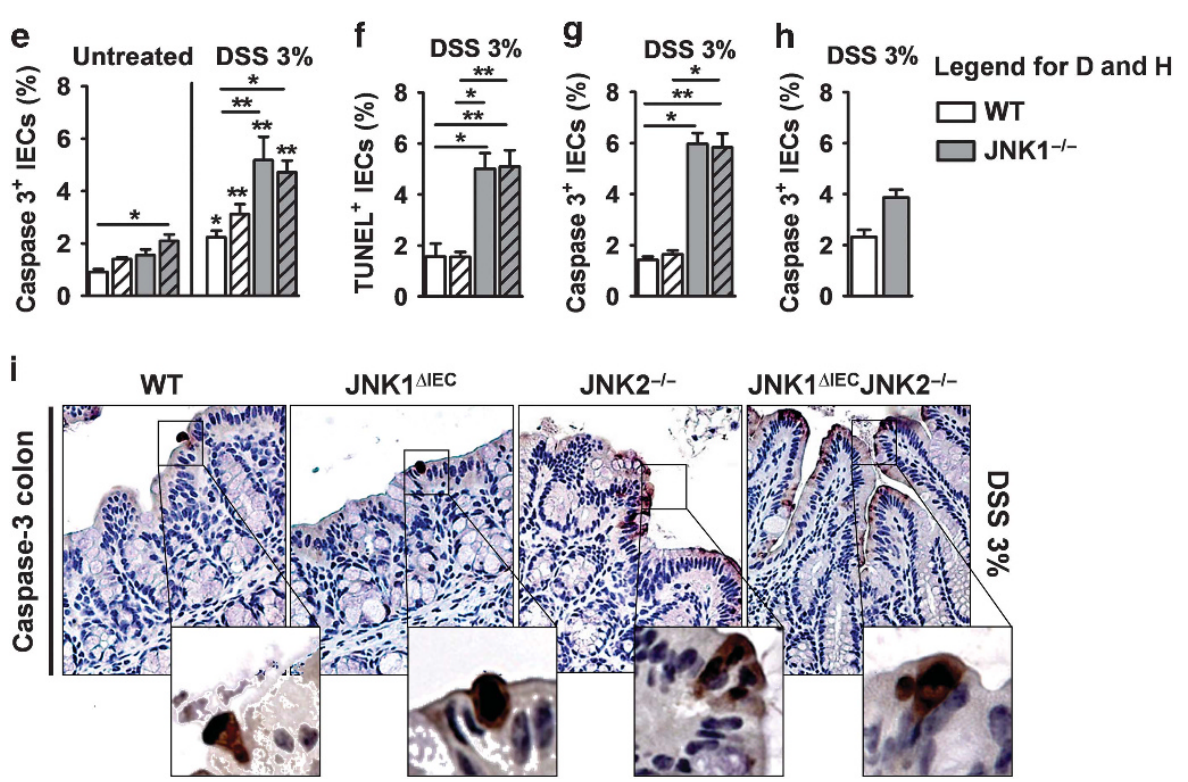

Figure 5 Increased intestinal epithelial cell (IEC) proliferation and apoptosis in Jun N-terminal kinase 2 (JNK2)-deficient mice. (a-d) Proliferating IECs were analyzed by bromodeoxyuridine (BrdU) labeling. BrdU were injected $2 \mathrm{~h}$ before killing. (a) Immunofluorescence (IF) for BrdU (green), phalloidin staining for F-actin (red), and DAPI (4',6-diamidino-2-phenylindole) staining for nuclei on colon sections from WT, JNK1 ${ }^{\Delta \mathrm{IEC}}$, JNK2 ${ }^{-1-}$, and JNK1 ${ }^{\Delta I E C}$ JNK2 $2^{-1-}$ mice at day 7 of dextran sulfate sodium (DSS) treatment is shown. Bar $=50 \mu \mathrm{m}$. (b-d) Quantification of BrdU-positive colonic IECs of indicated mice. (e-h) Apoptosis in IECs were analyzed by immunostaining for cleaved caspase-3 and TUNEL (terminal deoxynucleotidyl transferase dUTP nick-end labeling) assay. (e, $\mathbf{g}$, and $\mathbf{h}$ ) Percentage of cleaved caspase-3-positive IECs of indicated mice. (f) Percentage of TUNEL-positive IECs from 7 days DSS-treated WT, JNK1 ${ }^{\Delta \mathrm{IEC}}$, JNK2 ${ }^{-1-}$, and JNK1 ${ }^{\Delta \mathrm{IEC}} \mathrm{JNK} 2^{-1-}$ animals. (i) IHC for cleaved caspase-3 (brown) on colon sections from DSStreated WT, JNK $1^{\Delta \mathrm{EEC}}, \mathrm{JNK} 2^{-1-}$, and JNK1 $1^{\Delta \mathrm{IEC}} \mathrm{JNK} 2^{-1-}$ mice. Means \pm s.e.m. of $n=4-16$ per group are shown. Statistical analyses are performed as described for Figures 3c-f. ${ }^{\star} P<0.05 ;{ }^{* \star} P<0.01 ;{ }^{* \star} P<0.001$. Further representative images are shown in Supplementary Figures 7 and 8 . For negative controls, see Supplementary Figure 14. 
dependent on a JNK2 deficiency in the nonhematopoietic compartment (Figure 5g and Supplementary Figure 8C). In JNK1 $1^{\triangle \mathrm{IEC}}$ and JNK1 $1^{-1-}$ mice only a minor, not significant increase of IEC apoptosis was detectable (Figure 5e,f,h,i and Supplementary Figure 8A,B, and D).

To further investigate the influence of JNK2 on IEC development, proliferation, and apoptosis, we created IEC organoids from WT and JNK2 ${ }^{-1-}$ mice. The development of JNK2 ${ }^{-1-}$ organoids was significantly delayed (Figure 6a,b). In contrast to the in vivo situation, we found a slight, however, statistically not significant reduction of IEC proliferation in JNK2 ${ }^{-1-}$ organoids at day 7 after passage (Figure $6 \mathrm{c}$ ). In correlation with the in vivo results, enhanced expression of cleaved caspase- 3 indicating apoptotic IECs was found in JNK2 ${ }^{-1-}$ organoids in comparison with WT organoids (Figure 6d). Furthermore, FACS analysis revealed enhanced apoptosis in the human colon carcinoma cell line HT-29 after treatment with the pan-JNK inhibitor SP600125 (Figure 6e).

Taken together, these data suggest that JNK2 deletion increases the rate of IEC apoptosis rates, leading to secondary regenerative hyperproliferation in vivo.

\section{JNK1 and JNK2 control distinct transcriptional profiles during acute colitis}

At day 7 of DSS colitis, we analyzed transcriptional profiles by microarrays in colonic tissue of WT, JNK1 ${ }^{\mathrm{NK} 1}$, and JNK2 ${ }^{-/-}$ mice. Principal component analysis revealed major differences between the three genotypes (Figure 7a). Surprisingly, we found the highest number of differentially expressed genes by comparing JNK2 ${ }^{-l-}$ vs. JNK1 ${ }^{\mathrm{IEC}}$ (Supplementary Figure 9A and Supplementary Table 3). Some genes were inversely regulated in $\mathrm{JNK}_{1}^{\mathrm{IEC}}$ and $\mathrm{JNK} 2^{-1-}$ mice (Supplementary Figure 9B). Gene ontology enrichment analysis shows that JNK2 ${ }^{-1-}$ vs. WT differentially expressed genes preferentially participate in inflammation and stress response, whereas JNK1 $1^{\mathrm{IEC}}$ vs. WT regulated genes have a very different gene ontology profile with a strong enrichment in intracellular processes such as cellular component and nucleosome organization (Supplementary Figure 9C and Supplementary Table 3).

Enhanced stress response was also reflected by the increased expression of Bip and Xbp1_s by quantitative PCR (qPCR) in DSS-treated $\mathrm{JNK}_{2}{ }^{-1-}$ and $\mathrm{JNK} 1^{\mathrm{IEC}} \mathrm{JNK} 2^{-1-}$ mice, a
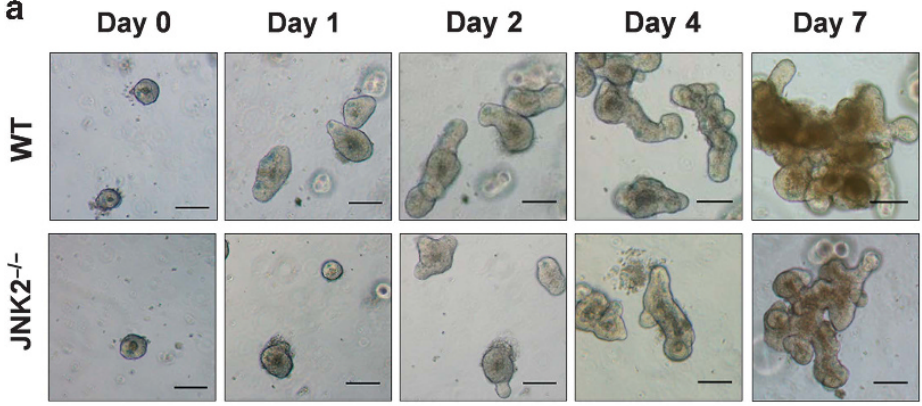

b

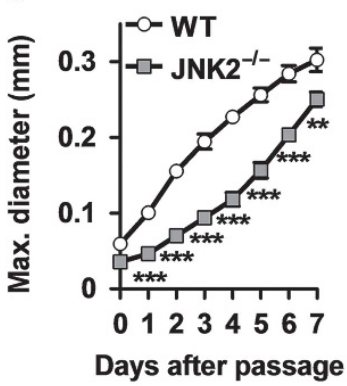

C
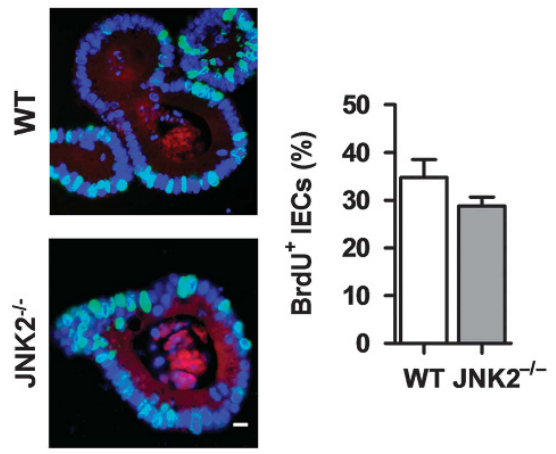

d

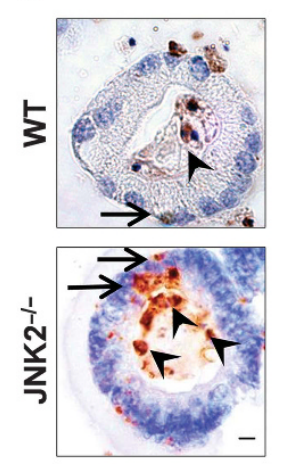

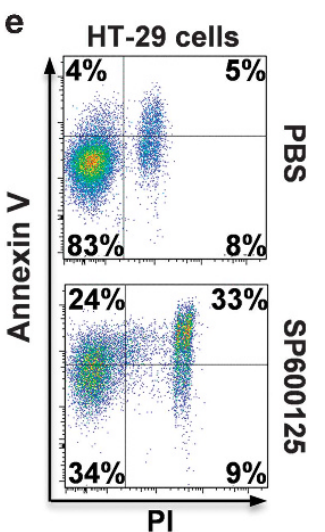

Figure 6 Increased apoptosis in JNK2 ${ }^{-1-}$ intestinal organoids and SP600125-treated HT-29 cells. (a) Small intestinal organoids were produced from wild-type (WT) and JNK2 ${ }^{-1-}$ crypt cells and passaged every 7 days. Representative images at indicated time points after passage are shown. (b) Maximum diameter of organoids at indicated time points after passage (mean \pm s.e.m., $n=30-45$ organoids per time point derived from three different experiments). (c) Immunofluorescence (IF) for bromodeoxyuridine (BrdU) (green), Phalloidin staining for F-actin (red) and DAPI (4',6-diamidino-2phenylindole) staining for nuclei in organoids. Graph represents percentage of BrdU ${ }^{+}$IECs in organoids (mean \pm s.e.m., $n=21$ (WT) and $n=35$ (JNK2 ${ }^{-1-}$ ) derived from three different experiments). (d) Immunostaining of cleaved caspase- $3^{+}$IECs (arrow) in organoids. Graph represents percentage of cleaved caspase- $3^{+}$IECs (mean \pm s.e.m., $n=15$ (WT) and $n=9$ (JNK2 ${ }^{-1-}$ ) derived from three different experiments). Apoptotic cells within the lumen and outside of the organoids may represent shed cells (arrowhead) and were not counted. Bar $=100 \mu \mathrm{m}(\mathbf{a})$ and $5 \mu \mathrm{m}$ (c and d). (e) HT-29 cells were treated with $25 \mu \mathrm{M}$ JNK inhibitor SP600125 for $24 \mathrm{~h}$ and apoptosis was analyzed by Annexin V and PI staining using flow cytometry. One representative FACS plot of three independent experiments is shown. Statistics was performed using the Mann-Whitney $U$-test. ${ }^{\star} P<0.05$; ${ }^{\star \star} P<0.01$; ${ }^{* * *} P<0.001$. IEC, intestinal epithelial cell; JNK, Jun $\mathrm{N}$-terminal kinase. 


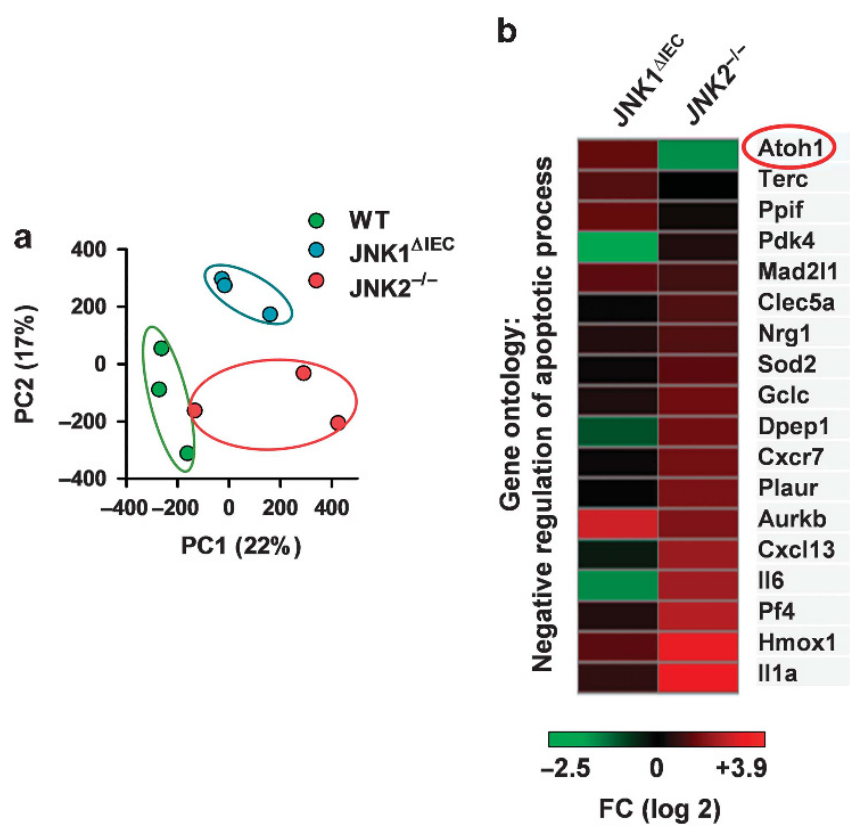

Figure 7 Wild-type (WT), JNK1 $1^{\triangle \mathrm{IEC}}$ and $\mathrm{JNK} 2^{-1-}$ mice have distinct transcriptional profiles. Affymetrix mRNA arrays were performed from colonic tissue of 7 days dextran sulfate sodium (DSS)-treated WT, $\mathrm{JNK} 1^{\triangle \mathrm{IEC}}$, and JNK2 ${ }^{-1-}$ mice ( $n=3$ per group). (a) Transcriptional profiles of different genotypes are discriminated by principle component analysis. The first two components indicating 22\% (PC1) and 17\% (PC2) of overall variability are shown. (b) Heat maps of genes classified in the GO category "negative regulation of apoptotic process" with a mean fold change (FC) of $\geq 2$ and statistical significance of $P<0.05$ in at least one comparison (JNK1 $1^{\mathrm{IEC}}$ vs. WT, JNK2 $2^{-1-}$ vs. WT or JNK1 $1^{\mathrm{IEC}}$ vs. JNK2 ${ }^{-1}$

-). Colors indicate $\mathrm{FC}$ of $\mathrm{JNK} 1^{\mathrm{IEC}}$ and $\mathrm{JNK} 2^{-1-}$ mice compared with WT animals. IEC, intestinal epithelial cell; JNK, Jun N-terminal kinase.

indicating enhanced endoplasmatic reticulum stress. However, these genes were not upregulated in untreated animals or intestinal $\mathrm{JNK}^{-1-}$ organoids, suggesting that elevated endoplasmatic reticulum stress is a consequence of higher inflammation and tissue destruction in DSS-treated JNK2 ${ }^{-1-}$ and $\mathrm{JNK} 1^{\mathrm{IEC}} \mathrm{JNK} 2^{-1-}$ mice (Supplementary Figure 10).

\section{JNK2 regulates Atoh1 and secretory cell regeneration}

Quantitative reverse transcription PCR analyses did not show differential expression of the apoptosis-related genes Bax, Bad, $B c l 2$, Tnfrsfla (TNF receptor 1), and Birc5 (Survivin) in DSStreated WT, JNK1 ${ }^{\triangle \mathrm{IEC}}, \mathrm{JNK} 2^{-/-}$, and JNK1 ${ }^{\triangle \mathrm{IEC}} \mathrm{JNK} 2^{-1-}$ mice. The proapoptotic factor Bak, known to be induced by $\mathrm{JNKs}^{26}{ }^{26}$ was downregulated in JNK2-deficient animals (Supplementary Figure 11A), which, however, cannot explain increased IEC apoptosis in these mice. The microarray data revealed that several genes with proapoptotic functions were significantly upregulated in $\mathrm{JNK}^{-I-}$ mice (Supplementary Figure 11B and C). However, many of these genes are associated with inflammation and tissue remodeling, suggesting that the elevated expression is probably a consequence of the increased inflammatory response in JNK2 ${ }^{-1-}$ animals and not a direct effect of deficient JNK2 signaling.
In the microarray analysis, Atonal homolog 1 (Atoh1) was the only significantly downregulated antiapoptotic factor in JNK2 ${ }^{-l-}$ mice (Figure 7b). Atoh1 is a transcription factor that is essential for goblet and enteroendocrine cell differentiation. ${ }^{27}$ qPCR analyses revealed that in untreated animals the Atoh 1 expression was downregulated in $\mathrm{JNK}_{1}{ }^{\triangle \mathrm{IEC}} \mathrm{JNK} 2^{-/-}$and slightly less importantly also in JNK2 ${ }^{-1-}$ mice in comparison with WT mice. During DSS colitis, Atoh1 expression was decreased in all four investigated genotypes compared with untreated mice, although this effect was more pronounced in $\mathrm{JNK} 2^{-1-}$ and JNK1 ${ }^{\triangle \mathrm{IEC}} \mathrm{JNK} 2^{-1-}$ mice (Figure 8a). Furthermore, we detected diminished expression of $\mathrm{Muc2}$, the major intestinal mucin, in $\mathrm{JNK} 2^{-1-}$ and $\mathrm{JNK} 1^{\triangle \mathrm{IEC}} \mathrm{JNK} 2^{-/-}$mice during DSS colitis. A tendency towards decreased $M u c 2$ expression was also found in untreated $\mathrm{JNK}^{-1-}$ and $\mathrm{JNK}^{\triangle \mathrm{IEC}} \mathrm{JNK}^{-1-}$ animals (Figure 8b). Concomitantly with diminished Atoh1 and Muc2 expression, we detected strongly decreased goblet cell numbers in DSS-treated $\mathrm{JNK}^{-I-}$ and JNK1 ${ }^{\triangle \mathrm{IEC}} \mathrm{JNK} 2^{-1-}$ mice, whereas no difference was observed in untreated animals (Figure 8c,d).

To exclude that the reduction of goblet cell numbers was only a consequence of increased inflammation and tissue destruction in JNK2 ${ }^{-1-}$ and $\mathrm{JNK}^{\triangle \mathrm{IEC}} \mathrm{JNK} 2^{-1-}$ mice, we further analyzed intestinal organoids. JNK2 ${ }^{-1-}$ organoids had $\sim 50 \%$ less goblet cells than WT organoids (Figure 8e). mRNA expression for Atoh1, Muc2, Muc5ac, and $M u c 5 b$ was reduced in $\mathrm{JNK}^{-1-}$ organoids (Figure 8f). mRNA expression for Atoh 1 and Muc2 was also downregulated in human HT-29 cells treated with the JNK inhibitor SP600125 (Figure 8g). We further found decreased numbers of chromogranin A-positive colonic enteroendocrine cells in JNK2-deficient mice during DSS colitis and in JNK2 ${ }^{-1-}$ organoids (Figure 9).

These results indicate that JNK2 regulates Atoh1 expression and secretory cell development. The JNK2 effect on secretory cells may be compensated during homeostasis; however, during stress response and rapid cell turnover (as in organoids), goblet end enteroendocrine cell regeneration is delayed in the absence of JNK2.

To understand the possible links between activity of JNK2 and expression of the mouse Atoh 1 gene, we have analyzed the signal-transduction and gene regulatory network using geneXplain platform (http: //www.genexplain.com) ${ }^{28}$ and TRANSFAC database. ${ }^{29}$ According to TRANSFAC, the mouse Atoh 1 gene has a well-studied enhancer in the $3^{\prime}$ region of the gene (Supplementary Figure 13) with multiple sites for several transcription factors. Among them we found five (LEF-1 (Lef1), Msx-1 (Msx1), E47/E12 (Tcf3), Sox-2 (Sox2), and MATH1 (Atoh1)) whose expression was observed to be changed in the JNK2 ${ }^{-l-}$ mice compared with WT mice. Sox-2 (Sox2), LEF-1 (Lef1), and E47/E12 (Tcf3) were upregulated and MATH1 (Atoh1) and Msx-1 (Msx1) were downregulated. We think that these transcription factors acting on their binding sites in the enhancer contribute collectively to the downregulation of Atoh1 gene. Further, we found possible links between these five transcription factors and JNK2 kinase using the masterregulator search engine of geneXplain platform. It searches for 

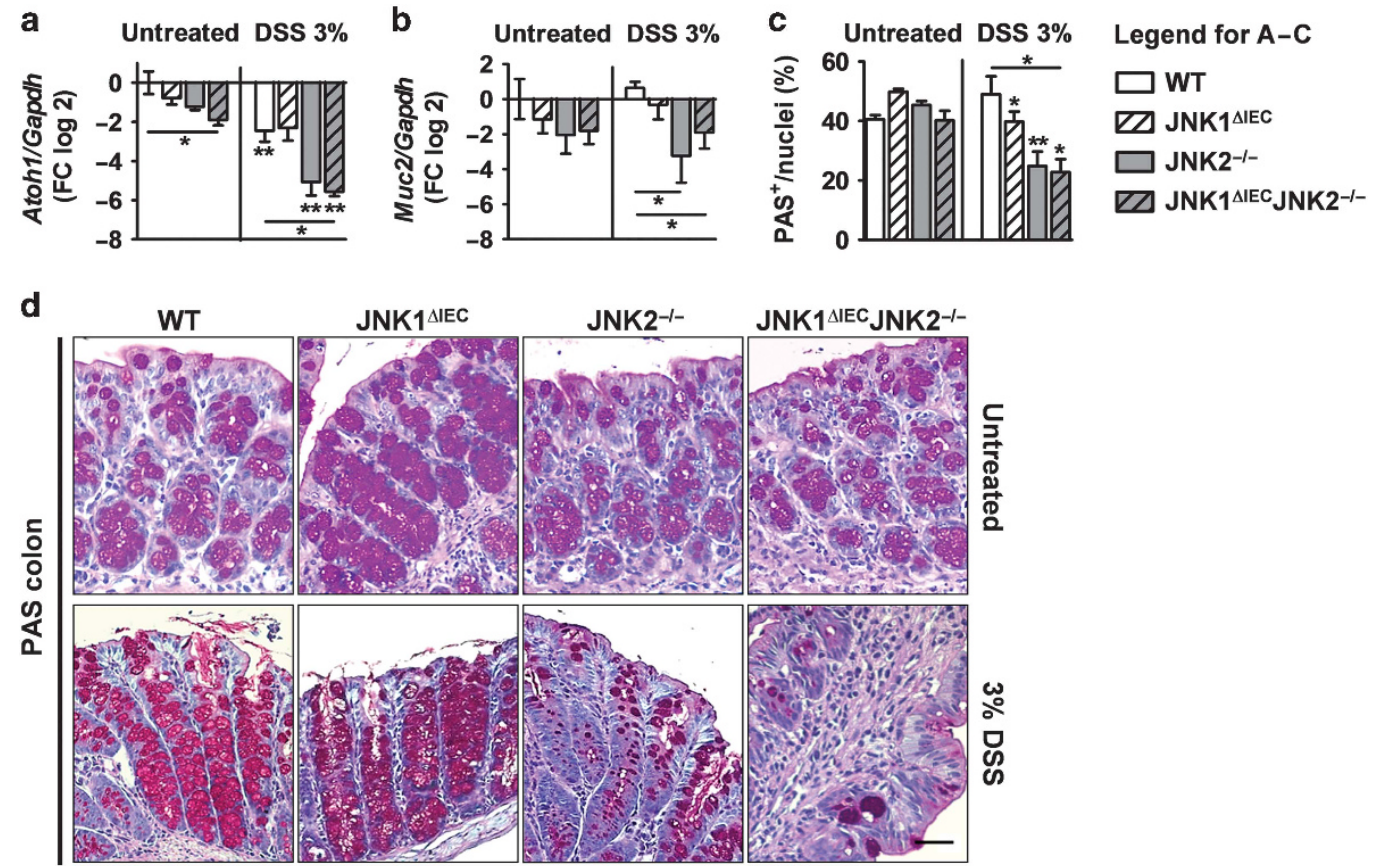

e

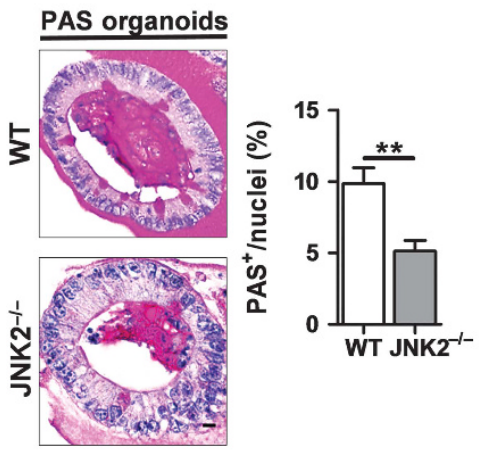

$\mathbf{f}$
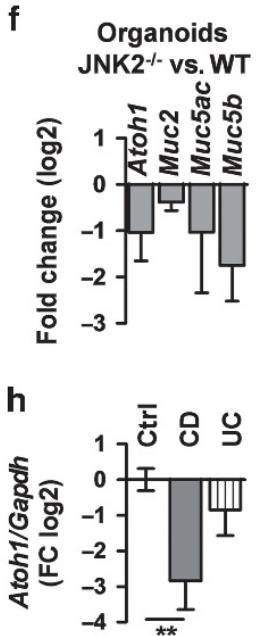

9 SP600125 vs. Con

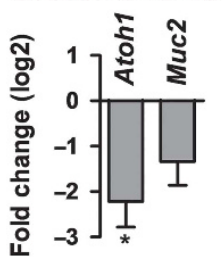

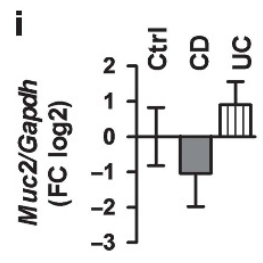

Figure 8 Jun N-terminal kinase (JNK2) regulates Atonal homolog 1 (Atoh1) expression and goblet cells regeneration. (a) Atoh1 and (b) Muc2 mRNA expression in colonic tissue derived from untreated and 7 days dextran sulfate sodium (DSS)-treated wild-type (WT), JNK1 $1^{\mathrm{IEC}}$, JNK2 ${ }^{-1-}$, and JNK1 ${ }^{\mathrm{IEC}} \mathrm{JNK} 2^{-1-}$ mice. Data are expressed as mean \pm s.e.m. of $\log 2$ fold change (FC) compared with the mean of untreated WT animals $(n=4-9)$. (c) Graphs represent percentage of Periodic Acid Schiff (PAS) ${ }^{+}$epithelial cells from untreated and 7 days DSS-treated mice of indicated genotypes (mean \pm s.e.m., $n=4-8$ ). (d) PAS staining of representative colon sections. (e) PAS staining of WT and JNK2 ${ }^{-/-}$organoids. Bar $=(\mathbf{d}) 20 \mu \mathrm{m}$ and $(\mathbf{e})$ $5 \mu \mathrm{m}$. Graphs represent percentage of PAS ${ }^{+}$IECs (mean \pm s.e.m., $n=15$ organoids derived from three different experiments). PAS staining within the lumen and outside the organoid is probably secreted mucus. (f) mRNA expression of Atoh1, Muc2, Muc5ac, and Muc5b in intestinal organoids. Gene expression is normalized to Gapdh expression and data are expressed as log 2 fold change (FC) comparing JNK2 ${ }^{-1-}$ vs. WT (mean \pm s.e.m.; $n=3$ ). (g) mRNA expression of Atoh 1 and Muc2 in HT29 cells after $3 \mathrm{~h}$ treatment with SP600125 in comparison with untreated HT29 cells (mean \pm s.e.m.; $n=5)$. (h) Atoh1 and (i) Muc2 mRNA expression in colonic tissue biopsies derived from patients with Crohn's disease (CD, $n=14)$, ulcerative colitis (UC; $n=11)$ and non-IBD controls (Ctrl; $n=14$ ). Statistics were performed using the Mann-Whitney U-test or Kruskal-Wallis test and Dunn's post hoc analysis (for (a)-(c) see detailed description in Figures $3 \mathbf{c}-\mathrm{f})$. ${ }^{\star} P<0.05 ;{ }^{\star *} P<0.01$. IEC, intestinal epithelial cell.

the shortest paths through the signal-transduction network of TRANSPATH database ${ }^{30}$ from the query transcription factors to the upstream regulators of the activity of the transcription factors. Through implementing a special graph weighting algorithm, ${ }^{28}$ it favors those of the paths through the network that go through signaling proteins whose expression is also changed in the JNK2 $2^{-1-}$ mice compared with WT mice. As a result, we have reconstructed the possible links between JNK2 kinase and five transcription factors that may explain the mechanism of the downregulation of Atoh1 expression in JNK2-knockout mice. Interestingly, as Atoh1 gene expresses the ATOH1/MATH1 transcription factor, we could also propose the autocatalytic downregulation mechanism, when upon JNK2 knockout the respective signaling reaches the 
a
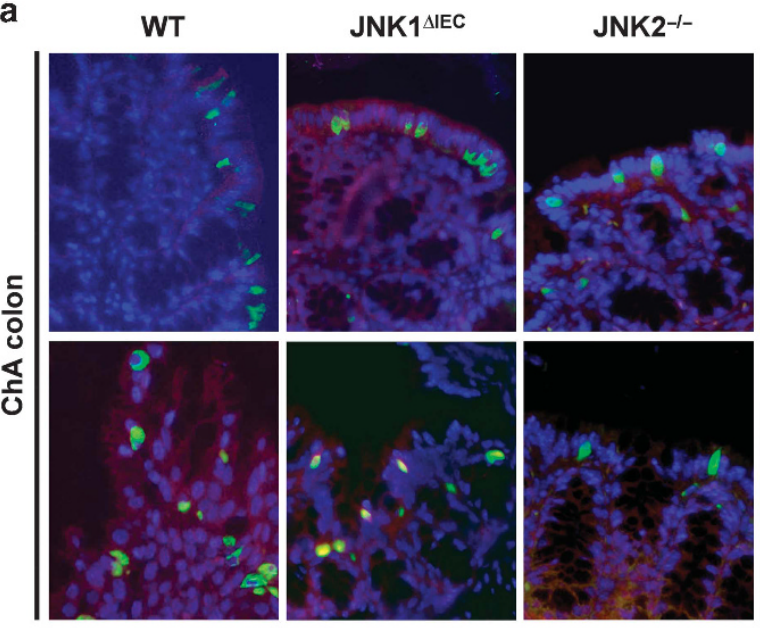

JNK1 $^{\triangle I E C}$ JNK2-I-
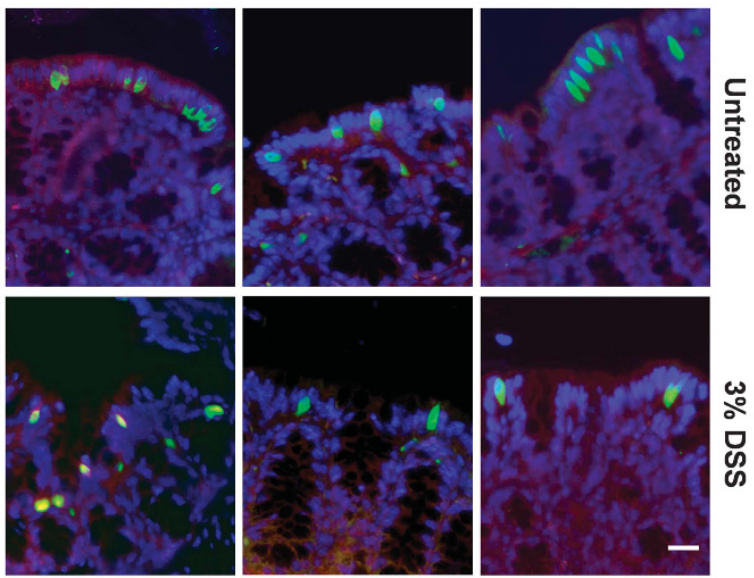

b

ChA
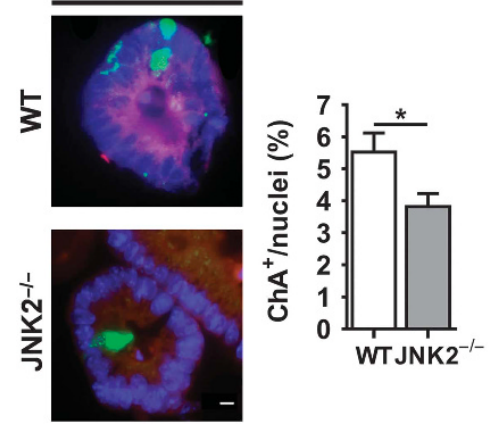

C

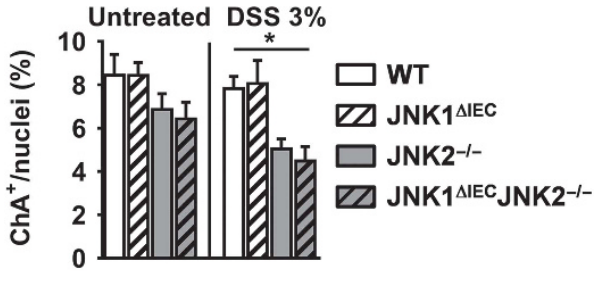

Figure 9 Jun N-terminal kinase (JNK2) regulates enteroendocrine cells differentiation. (a) Chromogranin A (ChA) staining of colon sections from untreated and 7 days dextran sulfate sodium (DSS)-treated mice of indicated genotypes. (b) ChA staining of wild-type (WT) and JNK2 ${ }^{-1-}$ organoids. $\mathrm{Bar}=$ (a) $10 \mu \mathrm{m}$ and (a) $5 \mu \mathrm{m}$. For negative control see Supplementary Figure 14. (c) Graphs represent percentage of ChA ${ }^{+}$intestinal epithelial cells (IECs) in organoids (mean \pm s.e.m., $n=15$ organoids derived from three different experiments) and from indicated mice (mean \pm s.e.m., $n=4-7$ ). Statistics were performed using the Mann-Whitney U-test or Kruskal-Wallis test and Dunn's post hoc analysis. ${ }^{*} P<0.05$.

Prox-1-the inhibitor of MATH1. The activity of MATH1 on the Atoh1 enhancer is decreased, which triggers the downregulation of the Atoh1 gene, which in turn decreases the expression of ATOH1/MATH1 protein, leading to the double effect of the repression.

Finally, we analyzed Atoh1 and Muc2 mRNA expression in tissue biopsies from the distal colon of IBD and non-IBD control patients. Atoh1 expression was significantly decreased in $\mathrm{CD}$ patients compared with controls, whereas it was not significantly different in UC patients (Figure 8h). Muc2 expression was not significantly different between the groups (Figure 8i); however, Muc2 expression correlated with the Atoh1 transcription levels in $\mathrm{CD}$ patients (Supplementary Figure 13A). Atoh1 expression levels were highly diverse in individual CD patients and were independent of inflammation (Supplementary Figure 13B).

\section{DISCUSSION}

In our present work, we identify a protective role of epithelial JNK2 signaling to maintain mucosal barrier function, epithelial cell integrity, and mucus layer production in the event of inflammatory tissue damage. In particular, we showed that
JNK2 prevents IEC apoptosis and promotes the expression of Atoh 1 and goblet and enteroendocrine cell regeneration.

All JNK-deficient mice used in our study developed normally and did not show disruption of the intestinal barrier or immune homeostasis. However, JNK2 $2^{-1-}$ mice showed more severe inflammation, tissue destruction, and weight loss after induction of acute DSS colitis, whereas JNK1-deficient mice had only a minor phenotype that, however, did not reach significance in our statistical analyses. These results confirmed previous results describing similar phenotypes in a model of chronic DSS colitis. ${ }^{24}$ We could demonstrate that JNK2 expression in nonhematopoietic cells, probably IECs, confers protections during acute DSS colitis. Mice with a combined JNK1/2 deficiency in IECs $\left(\mathrm{JNK}^{\mathrm{IEC}} \mathrm{JNK} 2^{-/-}\right.$) showed no significant differences in the colitis phenotype compared with JNK2 ${ }^{-1-}$ animals. These results reveal that JNK2 mediates protective functions in acute intestinal inflammation and that JNK1 cannot substitute for the loss of JNK2. The different function of JNK1 and JNK2 in IECs is further underlined by their distinct downstream effects, as we found that $\mathrm{p}$-c-Jun and $\mathrm{p}$-JunD expression was more affected in JNK1-deficient IECs than in JNK2-deficient IECs and that the transcriptional profiles were largely different in $\mathrm{JNK}^{\mathrm{IEC}}$ vs. JNK2 $2^{-/-}$animals. 
IEC turnover, apoptosis, and proliferation was increased in untreated as well as DSS-treated JNK2-deficient mice. The presence of ileal villus shortening in JNK2-deficient mice suggests a regenerative response with primarily elevated apoptosis, ${ }^{31}$ because primary hyperproliferation has been linked to villus elongation. ${ }^{19}$ This conclusion is further supported by the fact that IECs in JNK2 ${ }^{-1-}$ organoids showed higher rates of IEC apoptosis compared with IECs in WT organoids, whereas IEC proliferation was not significantly different. Furthermore, apoptosis was elevated in HT-29 cells treated with a JNK inhibitor.

Our data are in line with a recent report showing that $\mathrm{JNK}^{-1-}$ mice have elevated proliferation of ileal IECs. ${ }^{32}$ However, another publication reported unaltered small intestinal and colonic EC proliferation in JNK2-deficient mice during homeostasis. ${ }^{33}$ The reason for these different results remains elusive, while possibly environmental factors such as the specific microbiota in the local animal facilities have a role.

IEC-specific deletion of the main JNK downstream transcription factor c-Jun has been shown to cause decreased small intestinal stem cell proliferation, whereas activation of c-Jun by overexpression of constitutive active JNK1 was associated with enhanced IEC proliferation and tumorigenicity. ${ }^{19}$ The effect of c-Jun was shown to depend on a cross-talk with the Wnt/ $\beta$ catenin pathway via direct effects of c-Jun on Tcf4 and Lgr5 expression. ${ }^{18,19}$ In our experiments, phosphorylated c-Jun was strongly decreased, but not absent in colonic IECs of JNK $1^{\mathrm{IEC}}$ JNK2 ${ }^{-1-}$ mice; however, $\mathrm{p}$-c-Jun levels were comparable in $\mathrm{WT}$ and JNK2 ${ }^{-1-}$ mice. We found a tendency towards slightly reduced expression of $\mathrm{Wnt} / \beta$-catenin-dependent genes in the colon but not in the ileum of JNK-deficient animals, which however did not correlate with decreased colonic IEC proliferation. These results suggest that the observed phenotype, which was similar in JNK2 ${ }^{-1-}$ and JNK1 $1^{\mathrm{IEC}} \mathrm{JNK} 2^{-1-}$ mice, was not essentially dependent on $\mathrm{p}$-c-Jun or $\mathrm{Wnt} / \beta$ catenin-dependent pathways.

By analyzing the expression of antiapoptotic genes by mRNA microarray analysis, we observed a significant downregulation of Atoh1 expression in JNK2 $2^{-1-}$ mice during DSS colitis. Quantitative reverse transcription PCR analyses confirmed this result and reduced Atoh1 expression was also found in JNK2deficient mice during homeostasis as well as in $\mathrm{JNK}^{-1-}$ organoids and in human JNK inhibitor-treated HT-29 cells. Atoh1 is a transcription factor that regulates the differentiation of all secretory cell lineages in the intestine. ${ }^{27}$ Furthermore, Atoh1 expression is often reduced in colon carcinomas and has been described as a tumor suppressor gene. ${ }^{34}$ Concomitant with lower Atoh1 expression, we found reduced Muc2 expression and decreased goblet and enterendocrine cell numbers in DSS-treated JNK2-deficient mice and JNK2 ${ }^{-1-}$ organoids, whereas goblet and enterendocrine cell numbers were not significantly altered in untreated JNK2-deficient animals. These results suggest that secretory cell growth is particularly affected by JNK2 in situations with a high cell turnover as it occurs in regenerative responses. Goblet cell functions and MUC2 production are important for the maintenance of the gastrointestinal barrier and the protection against spontaneous colitis. $^{35}$ Therefore, we identified that the control of Atoh1 expression and goblet cell regeneration is an important mechanisms for the protective functions of JNK2 in DSS colitis.

We found decreased Atoh 1 colonic mRNA expression in CD but not in UC patients compared with controls. Furthermore Atoh1 and Muc2 showed a strong correlation in particular in CD patients, confirming earlier publications. ${ }^{36,37}$ In contrast to our results, recent reports found a decrease of Atoh1 expression in UC but not in CD patients. ${ }^{36,38}$ One possible explanation might be that Atoh1 mRNA expression is only affected in the subgroup of IBD patients who was overrepresented in our CD cohort and underrepresented in our UC cohort. This hypothesis is also supported by the fact that Atoh 1 mRNA expression levels were very heterogeneous in our CD patients, which however was independent of the presence of active disease. Additional analyses in larger cohorts are required for more detailed subgroup analyses and further evaluation of the association of Atoh1 expression and JNK activity.

One of our initial hypotheses was that cell-type-specific functions and compensatory mechanisms of JNK1 and JNK2 may explain some of the conflicting results that have been reported in experimental colitis models using either pharmacological inhibitors or single knockout mice for either JNK1 or JNK2. However, none of our JNK knockout mice showed an improved colitis phenotype as it has been reported for the panJNK inhibitor SP600125 (refs 3,20,22 and the JNK1-specific inhibitor XG-102. ${ }^{23,39}$ There might be four possible explanations that are not mutually exclusive: (i) Inhibitors may only partially block JNKs, thereby inhibiting proinflammatory effects while maintaining IEC cytoprotective functions. (ii) A total inhibition of all JNKs in immune cells may be required to constitute anti-inflammatory effects. However, we have not tested a combined JNK1/JNK2 knockout in immune cells. (iii) JNK inhibitors have off-target effects that mediate antiinflammatory and cytoprotective functions. Indeed, SP600125 targets several other kinases, although higher concentrations are required. ${ }^{40}$ (iv) Genetic deletions lead to genetic compensation that might not be the case after pharmacological inhibition. ${ }^{41}$ Further studies are required to fully understand the different effects of pharmacological inhibitors and genetic deletions of JNK in experimental models of intestinal inflammation.

In summary, we identify JNK2 as an important antiapoptotic factor for IECs and inducer of goblet and enteroendocrine cell regeneration. Our study provides a link to some of the recently identified mitogen-activated protein-kinase-related genetic variations in IBD patients ${ }^{6}$ and helps to further understand the role of central signalling pathways in gut homeostasis and inflammation.

\section{METHODS}

Human material. Colon biopsies were obtained from IBD and control patients during routine colonoscopy at the University Hospital RWTH Aachen. For IHC samples were fixed in $4 \%$ paraformaldehyde and embedded in paraffin, and for qPCR analyses, samples were 
immediately snap frozen in liquid nitrogen and stored at $-80^{\circ} \mathrm{C}$. Patients provided written informed consent. The study was approved by the local ethics committee (reference number EK 049/12).

Generation of knockout mice and BMT. Animals were housed in the University Hospital RWTH Aachen animal facility. The study was approved by the local ethics committee (approval reference number 87-51.04.201.A153). JNK1 ${ }^{\text {loxP/loxP } 42}$ JNK2 ${ }^{-}{ }^{-}$mice, ${ }^{43}$ and Villin-Cre mice $^{44}$ were crossed to create JNK1 $1^{\text {loxP/loxP }}$ (WT genotype), $\mathrm{JNK}^{\text {loxP/loxP}} / \mathrm{Villin}^{-C r \mathrm{C}^{+}}$(JNK1 $\left.^{\Delta \mathrm{IEC}}\right), \quad \mathrm{JNK} 1^{\text {loxP/loxP}} / \mathrm{JNK} 2^{-/-}$ $\left(\mathrm{JNK} 2^{-1-}\right)$, and $\mathrm{JNK} 1^{\text {loxP/loxP}} /$ Villin-Cre ${ }^{+} / \mathrm{JNK} 2^{-/-}\left(\mathrm{JNK} 1^{\Delta \mathrm{IEC}}\right.$ JNK2 $\left.{ }^{-1-}\right)$. Constitutive JNK1 $1^{-1-}$ mice ${ }^{45}$ were compared with WT littermates. All animals were kept on a C57BL/6; 129 background. To create chimeric $\mathrm{WT} / \mathrm{JNK} 2^{-1-}$ mice, $1 \times 10^{6}$ bone marrow cells from WT or JNK2 ${ }^{-1-}$ donors were intravenously injected into 7 -week-old WT and JNK2 ${ }^{-1-}$ recipients after ablative $\gamma$-irradiation. Eight weeks after BMT, mice were used for further experiments. Genotypes, gene deletions after Cre recombination, and efficient BMT were confirmed by PCR of genomic DNA (Supplementary Figure 1 and data not shown; for primers see Supplementary Table 1).

Induction and evaluation of DSS colitis. Mice were treated with 3\% $\left(\mathrm{w} \mathrm{v}^{-1}\right)$ DSS (MP Biomedicals; Heidelberg, Germany; MW 40,000) in drinking water to induce acute colitis. DAI at days $0,3,5$, and 7 was determined by combining scores based on change in weight $(0:<0 \%$; 1: $0-5 \% ; 2: 5-10 \% ; 3: 10-15 \% ; 4:>15 \%)$, stool consistency (0: normal; 2: loose stools; 4 : diarrhea), and fecal blood ( 0 : normal; 2 : guaiac test + ; 4: gross bleeding) divided by $3 .{ }^{46}$ To quantify/detect proliferating cells, $30 \mathrm{mg} \mathrm{g}^{-1}$ of bromodeoxyuridine (BrdU) was intraperitoneally injected at day 7 . Animals were killed $2 \mathrm{~h}$ later and organs were harvested for further analyses.

Organoid culture. Crypts from small intestine were isolated from 11-week-old WT and JNK2 ${ }^{-1-}$ mice (three mice per group) by incubation for $30 \mathrm{~min}$ at $4{ }^{\circ} \mathrm{C}$ in phosphate-buffered saline containing 2 mM EDTA as described previously. ${ }^{47}$ Isolated crypts were embedded in Matrigel (growth factor reduced, phenol red free; BD Biosciences, Franklin Lakes, NJ) and seeded into 48 -well plates $(20 \mu \mathrm{l}$ of Matrigel per well). The Matrigel was polymerized for $15 \mathrm{~min}$ at $37^{\circ} \mathrm{C}$, and $250 \mu \mathrm{l}$ per well basal culture medium (advanced DMEM/F12 medium supplemented with penicillin/streptomycin, $10 \mathrm{mmoll}^{-1}$ HEPES, Glutamax, $1 \times \mathrm{N} 2,1 \times \mathrm{B} 27$ (all from Invitrogen, Carlsbad, CA), $1 \mathrm{mmol} \mathrm{l}^{-1} \mathrm{~N}$-acetylcysteine (Sigma-Aldrich, St. Louis, MO), murine EGF (Invitrogen), murine noggin (PeproTech, Hamburg, Germany), and a fusion protein between the Wnt agonist R-spondin-1 and immunoglobulin Fc (RSpo1-Fc). RSpo1-Fc was purified from the supernatant of stably transfected HEK293T cells as described recently. ${ }^{48}$ Fresh medium was added every 3 days and organoids were passaged after 7 days with a 1:5 split ratio. Organoids were monitored daily and length was calculated using the ImageJ software $(\mathrm{NIH}$, Bethesda, MD) and expressed as mean of at least 15 measured organoids per animal. Samples for IF, IHC, and RNA isolation were obtained at day 7 after passage.

Histopathology score, Periodic Acid Schiff staining, IHC, and immunofluorescence. Colons (without cecum) and small intestines from DSS-treated and control mice were removed and flushed with phosphate-buffered saline. The terminal $1.5 \mathrm{~cm}$ were retrieved and used for RNA isolation and MPO assay (see below). The remaining part was opened longitudinally, rolled up, and cut longitudinally in two pieces. One part was fixed with $4 \%$ paraformaldehyde and embedded in paraffin. The other part was embedded in TISSUE TEK O.C.T. (Dako, Jena, Germany) for cryosections. Intestinal organoids were fixed in Carnoy overnight at $4{ }^{\circ} \mathrm{C}$ and embedded in paraffin.

Paraffin-embedded tissue sections from human biopsies and mice and mouse intestinal organoids were deparaffinized, rehydrated, and subjected to hemotoxylin and eosin staining, Periodic Acid Schiff staining, or IHC. A histopathology score was determined semiquantitatively by the sum of the following criteria: (i) epithelial damage, (ii) loss of goblet cell, (iii) inflammatory cell infiltration, and (iv) submucosa thickening (for (i); 0: normal; 2: irregular epithelial cell morphology in $<1 / 3$ of total area; 4 : irregular epithelial cell morphology in $>1 / 3$ of total area and/or small erosions; 6 : presence of ulcers with $<10 \%$ of ulcerated areas; $8: 10-20 \%$ of ulcerated areas; 10: $>20 \%$ of ulcerated areas); for ii-iv; 0 : normal; 1 : mild; 2 : moderate; 3: severe). Length of ileal villi was analyzed in areas with optimal cutting orientation using the ImageJ software and expressed as mean of at least 20 measured villi per animal. Goblet cells were quantified by Periodic Acid Schiff staining.

For IHC of mouse and human samples, sections were subjected to antigen retrieval and incubated overnight at $4{ }^{\circ} \mathrm{C}$ with primary antibodies (Abs) against cleaved caspase- 3 , lysozyme $\mathrm{C}$, chromogranin A or p-JNK diluted in Tris-buffered saline/bovine serum albumin 1\%/ Tween $0.1 \%$ (see Supplementary Table 2 for details of all Abs used in this study). As secondary antibody biotinylated goat anti-rabbit immunoglobulin $\mathrm{G}$ or conjugated with FITC were used and incubated for $2 \mathrm{~h}$ at $20^{\circ} \mathrm{C}$, followed by incubation with streptavidin-ABCalkaline phosphatase, color development with the Vector Red Staining Kit (both Vector Laboratories, Lörrach, Germany) and hematoxylin counterstaining.

Colon cryosections were stained with Abs against CD11b, BrdU, and zonula occludens protein-1. Secondary antibodies conjugated with FITC or Cy3 were used. An Alexa Fluor 594 phalloidin probe (Life Technologies, Carlsbad, CA) was used for F-actin staining in samples stained with FITC Abs. TUNEL (terminal deoxynucleotidyl transferase dUTP nick-end labeling) assays were performed on cryosections according to the manufacturer's instructions (In Situ Cell Death Detection Kit, Fluorescein; Roche, Basel, Switzerland). Mounting solution containing DAPI (4',6-diamidino-2-phenylindole; Vector Laboratories) was used to counterstain nuclei.

Statistics. Data are expressed as means ( \pm s.e.m.) or medians ( \pm interquartile ranges) as indicated. Statistical significance was analyzed by Mann-Whitney $U$-test for comparison of two groups and by Kruskal-Wallis test followed by Dunn's post hoc test for the comparison of multiple groups. Correlation was performed by using the Spearman's correlation coefficient $\left(r_{\mathrm{s}}\right)$. Statistical analyses were performed using the GraphPad Prism (La Jolla, CA).

SUPPLEMENTARY MATERIAL is linked to the online version of the paper at http://www.nature.com/mi

\section{ACKNOWLEDGMENTS}

We thank Andrea Puhar and Christian Liedtke for discussion, careful reading of the manuscript, and insightful comments, Ursula Schneider for excellent technical assistance, Florian Greten for providing Villin-Cre mice and Calvin Kuo for providing RSpo1-Fc-producing HEK cells. The work was supported by the START program of the faculty of medicine, RWTH Aachen (START 25/11), the German Research Foundation (DFG SE 1122/1 and SFB985/C3), and the European Union's Seventh Framework Programme (FP7/2007-2013) under grant agreement n 305564 (SysmedIBD).

\section{AUTHOR CONTRIBUTIONS}

Conceived and designed the experiments: A.M., R.D., F.C., C.T., and G.S. Performed the experiments: A.M., E.B., J.V., and S.R. Organoid culture: K.Z. and MH. Microarrays: B.D. Bioinformatic analysis: A.K. and G.S. General data analysis: A.M. and G.S. Collected patients samples: K.S. and G.S. Wrote the paper: A.M., M.H., F.C., and G.S.

\section{DISCLOSURE}

The authors declared no conflict of interest.

c 2017 Society for Mucosal Immunology 


\section{REFERENCES}

1. Hommes, D. et al. Inhibition of stress-activated MAP kinases induces clinical improvement in moderate to severe Crohn's disease. Gastroenterology 122, 7-14 (2002).

2. Bantel, H., Schmitz, M.L., Raible, A., Gregor, M. \& Schulze-Osthoff, K. Critical role of NF-kappaB and stress-activated protein kinases in steroid unresponsiveness. FASEB J. 16, 1832-1834 (2002).

3. Mitsuyama, K. et al. Pro-inflammatory signaling by Jun-N-terminal kinase in inflammatory bowel disease. Int. J. Mol. Med. 17, 449-455 (2006).

4. Roy, P.K., Rashid, F., Bragg, J. \& Ibdah, J.A. Role of the JNK signal transduction pathway in inflammatory bowel disease. World J. Gastroenterol. 14, 200-202 (2008).

5. Cannons, J.L., Hoeflich, K.P., Woodgett, J.R. \& Watts, T.H. Role of the stress kinase pathway in signaling via the T cell costimulatory receptor 41BB. J. Immunol. 163, 2990-2998 (1999).

6. Jostins, L. et al. Host-microbe interactions have shaped the genetic architecture of inflammatory bowel disease. Nature 491, 119-124 (2012).

7. Kim, J.Y., Omori, E., Matsumoto, K., Nunez, G. \& Ninomiya-Tsuji, J. TAK1 is a central mediator of NOD2 signaling in epidermal cells. J. Biol. Chem. 283, 137-144 (2008).

8. Haridas, V. et al. VEGl, a new member of the TNF family activates nuclear factor-kappa B and c-Jun N-terminal kinase and modulates cell growth. Oncogene 18, 6496-6504 (1999).

9. Salmeron, A., Ahmad, T.B., Carlile, G.W., Pappin, D., Narsimhan, R.P. \& Ley, S.C. Activation of MEK-1 and SEK-1 by Tpl-2 proto-oncoprotein, a novel MAP kinase kinase kinase. EMBO J. 15, 817-826 (1996).

10. Bogoyevitch, M.A., Ngoei, K.R., Zhao, T.T., Yeap, Y.Y. \& Ng, D.C. C-Jun $\mathrm{N}$-terminal kinase (JNK) signaling: recent advances and challenges. Biochim. Biophys. Acta 1804, 463-475 (2010).

11. Sabio, G. \& Davis, R.J. TNF and MAP kinase signalling pathways. Semin. Immunol. 26, 237-245 (2014).

12. Bogoyevitch, M.A. \& Kobe, B. Uses for JNK: the many and varied substrates of the c-Jun N-terminal kinases. Microbiol. Mol. Biol. Rev. 70, 1061-1095 (2006).

13. Dhanasekaran, D.N. \& Reddy, E.P. JNK signaling in apoptosis. Oncogene 27, 6245-6251 (2008).

14. Kuan, C.Y., Yang, D.D., Samanta Roy, D.R., Davis, R.J., Rakic, P. \& Flavell, R.A. The Jnk1 and Jnk2 protein kinases are required for regional specific apoptosis during early brain development. Neuron 22, 667-676 (1999).

15. Hess, P., Pihan, G., Sawyers, C.L., Flavell, R.A. \& Davis, R.J. Survival signaling mediated by $\mathrm{C}$-Jun $\mathrm{NH}(2)$-terminal kinase in transformed $\mathrm{B}$ lymphoblasts. Nat. Genet. 32, 201-205 (2002).

16. Hui, L., Zatloukal, K., Scheuch, H., Stepniak, E. \& Wagner, E.F. Proliferation of human HCC cells and chemically induced mouse liver cancers requires JNK1-dependent p21 downregulation. J. Clin. Invest. 118, 3943-3953 (2008).

17. Lamb, J.A., Ventura, J.J., Hess, P., Flavell, R.A. \& Davis, R.J. JunD mediates survival signaling by the JNK signal transduction pathway. Mol. Cell 11, 1479-1489 (2003).

18. Aguilera, C., Nakagawa, K., Sancho, R., Chakraborty, A., Hendrich, B. \& Behrens, A. C-Jun N-terminal phosphorylation antagonises recruitment of the Mbd3/NuRD repressor complex. Nature 469, 231-235 (2011).

19. Sancho, R. et al. JNK signalling modulates intestinal homeostasis and tumourigenesis in mice. EMBO J. 28, 1843-1854 (2009).

20. Samak, G., Chaudhry, K.K., Gangwar, R., Narayanan, D., Jaggar, J.H. \& Rao, R. Calcium/Ask1/MKK7/JNK2/C-Src signalling cascade mediates disruption of intestinal epithelial tight junctions by dextran sulfate sodium. Biochem. J. 465, 503-515 (2015).

21. Samak, G., Suzuki, T., Bhargava, A. \& Rao, R.K. c-Jun NH2-terminal kinase-2 mediates osmotic stress-induced tight junction disruption in the intestinal epithelium. Am. J. Physiol. Gastrointest. Liver Physiol. 299, G572-G584 (2010).

22. Assi, K., Pillai, R., Gomez-Munoz, A., Owen, D. \& Salh, B. The specific JNK inhibitor SP600125 targets tumour necrosis factor-alpha production and epithelial cell apoptosis in acute murine colitis. Immunology 118, 112-121 (2006).

23. Reinecke, K. et al. The JNK inhibitor XG-102 protects against TNBSinduced colitis. PLoS One 7, e30985 (2012).
24. Chromik, A.M. et al. Genetic deletion of JNK1 and JNK2 aggravates the DSS-induced colitis in mice. J. Invest. Surg. 20, 23-33 (2007).

25. Kersting, S. et al. Knockout of the C-Jun N-terminal Kinase 2 aggravates the development of mild chronic dextran sulfate sodium colitis independently of expression of intestinal cytokines TNFalpha, TGFB1, and IL-6. J. Inflamm. Res. 6, 13-23 (2013).

26. Fan, M., Goodwin, M.E., Birrer, M.J. \& Chambers, T.C. The c-Jun NH(2)terminal protein kinase/AP-1 pathway is required for efficient apoptosis induced by vinblastine. Cancer Res. 61, 4450-4458 (2001).

27. Yang, Q., Bermingham, N.A., Finegold, M.J. \& Zoghbi, H.Y. Requirement of Math1 for secretory cell lineage commitment in the mouse intestine. Science 294, 2155-2158 (2001).

28. Kel, A.E. et al. Multi-omics "Upstream Analysis" of regulatory genomic regions helps identifying targets against methotrexate resistance of colon cancer. EUPA Open Proteomics 13, 1-13 (2016).

29. Matys, V. et al. TRANSFAC and its module TRANSCompel: transcriptional gene regulation in eukaryotes. Nucleic Acids Res. 34, D108-D110 (2006).

30. Krull, M. et al. TRANSPATH: an information resource for storing and visualizing signaling pathways and their pathological aberrations. Nucleic Acids Res. 34, D546-D551 (2006).

31. Kaser, A. et al. XBP1 links ER stress to intestinal inflammation and confers genetic risk for human inflammatory bowel disease. Cell 134, 743-756 (2008).

32. Chung, H.K. et al. Jnk2 deletion disrupts intestinal mucosal homeostasis and maturation by differentially modulating RNA-binding proteins HuR and CUGBP1. Am. J. Physiol. Cell Physiol. 306, C1167-C1175 (2014).

33. Cellurale, C. et al. Role of JNK in mammary gland development and breast cancer. Cancer Res. 72, 472-481 (2012).

34. Bossuyt, W. et al. Atonal homolog 1 is a tumor suppressor gene. PLoS Biol. 7, e39 (2009).

35. Van der Sluis, M. et al. Muc2-deficient mice spontaneously develop colitis, indicating that MUC2 is critical for colonic protection. Gastroenterology 131, 117-129 (2006).

36. Gersemann, M. et al. Differences in goblet cell differentiation between Crohn's disease and ulcerative colitis. Differ. Res. Biol. Divers. 77, 84-94 (2009).

37. Zheng, X. et al. Suppression of hath1 gene expression directly regulated by hes 1 via notch signaling is associated with goblet cell depletion in ulcerative colitis. Inflamm. Bowel Dis. 17, 2251-2260 (2011).

38. Gersemann, M. et al. Olfactomedin-4 is a glycoprotein secreted into mucus in active IBD. J. Crohn's Colitis 6, 425-434 (2012).

39. Kersting, S. et al. The impact of JNK inhibitor D-JNKI-1 in a murine model of chronic colitis induced by dextran sulfate sodium. J. Inflamm. Res. 6, 71-81 (2013).

40. Bennett, B.L. et al. SP600125, an anthrapyrazolone inhibitor of Jun N-terminal kinase. Proc. Natl Acad. Sci. USA 98, 13681-13686 (2001).

41. Rossi, A. et al. Genetic compensation induced by deleterious mutations but not gene knockdowns. Nature 524, 230-233 (2015).

42. Das, M. et al. Suppression of p53-dependent senescence by the JNK signal transduction pathway. Proc. Natl Acad. Sci. USA 104, 15759-15764 (2007)

43. Yang, D.D. et al. Differentiation of CD4 + T cells to Th1 cells requires MAP kinase JNK2. Immunity 9, 575-585 (1998).

44. Madison, B.B., Dunbar, L., Qiao, X.T., Braunstein, K., Braunstein, E. \& Gumucio, D.L. Cis elements of the villin gene control expression in restricted domains of the vertical (crypt) and horizontal (duodenum, cecum) axes of the intestine. J. Biol. Chem. 277, 33275-33283 (2002).

45. Dong, C., Yang, D.D., Wysk, M., Whitmarsh, A.J., Davis, R.J. \& Flavell, R.A Defective T cell differentiation in the absence of Jnk1. Science 282, 2092 2095 (1998).

46. Murthy, S.N., Cooper, H.S., Shim, H., Shah, R.S., Ibrahim, S.A. \& Sedergran, D.J. Treatment of dextran sulfate sodium-induced murine colitis by intracolonic cyclosporin. Dig. Dis. Sci. 38, 1722-1734 (1993).

47. Sato, T. et al. Single Lgr5 stem cells build crypt-villus structures in vitro without a mesenchymal niche. Nature 459, 262-265 (2009).

48. Ootani, A. et al. Sustained in vitro intestinal epithelial culture within a Wntdependent stem cell niche. Nat. Med. 15, 701-706 (2009). 